\title{
A Weak Stochastic Integral in Banach Space with Application to a Linear Stochastic Differential Equation*
}

\author{
Nadav Berman ${ }^{\mathrm{I}}$ and William L. Root ${ }^{2}$ \\ ${ }^{1}$ Department of Aeronautical Engineering, Technion, Haifa, 32000 Israel, and ${ }^{2}$ Aerospace Engineering \\ Department, The University of Michigan, Ann Arbor, MI 48109 \\ Communicated by A. V. Balakrishnan
}

\begin{abstract}
Cylindrical Wiener processes in real separable Banach spaces are defined, and an approximation theorem involving scalar Wiener processes is given for such processes. A weak stochastic integral for Banach spaces involving a cylindrical Wiener process as integrator and an operator-valued stochastic process as integrand is defined. Basic properties of this integral are stated and proved.

A class of linear, time-invariant, stochastic differential equations in real, separable, reflexive Banach spaces is formulated in such fashion that a solution of the equation is a cylindrical process. An existence and uniqueness theorem is proved. A stochastic version of the problem of heat conduction in a ring provides an example.
\end{abstract}

\section{Introduction}

Stochastic integrals and stochastic differential equations in abstract spaces have been of interest for some time, see, e.g., [3, 6, 7, 11, 12, 15, 16, 17, 18]. Most, but not all, of the work in this area has had to do with "proper" stochastic processes taking values in a Hilbert or Banach space, i.e., processes $\left\{X_{t}, t \in T \subset R\right\}$ such that $X_{t}$ is a (say, strongly) measurable function on a probability space. We are concerned here, however, with "weak" stochastic processes which are associated with (finitely-additive) cylinder set probability measures. Cylindrical stochastic processes (c.s.p.) as defined in Section 2 below are such processes, but to avoid

${ }^{*}$ Research supported by National Science Foundation under Grant No. ECS- 8005960 . 
involvement with various technically different definitions we continue to use the term, weak process, in a somewhat vague sense in these introductory remarks.

In Chapters 3 and 4 of [3], Bensoussan introduced weak processes and used them to model white noise forcing functions in an investigation of a general type of linear stochastic equation of evolution in Hilbert space. The method of solution employed by Bensoussan is to determine an affine "input-state" map using theory for deterministic differential equations, and then apply the map to the input white noise. No stochastic integration theory as such is necessary for this development, although "strong" stochastic integrals in Hilbert space are defined and used elsewhere in [3]. Balakrishnan, [1], has also used white noise based on cylinder set measures in a treatment of linear stochastic equations of evolution in Hilbert space. In both of these references the theory is applied to the filtering problem for linear dynamical systems.

Stochastic integrals in Hilbert space have been widely discussed and used, but there is an inherent difficulty in extending stochastic integration in the ordinary sense to Banach space. This fact is noted in the book [16] of Metivier and Pellaumail (see Sect. 16), and they define a weak-sense stochastic integral in Banach space to circumvent the difficulty. Their integral is defined for a class of integrator processes ("2-cylindrical martingales with finite quadratic variation").

Since ordinary stochastic integration theory does not work properly in Banach space (and in particular, in the case where the integrator is a Wiener process) there is considerable motivation to study weak integrals and their application to differential equations. Even in the Hilbert-space case there are good reasons for using weak processes (and hence, it would appear, weak integrals) in certain applications, as is adequately pointed out in [1] and [3].

In this paper we first define a cylindrical Wiener process (c.W.p.) in a real separable Banach space $B$ and get a representation (Theorem 2.2) that shows such a process can always be obtained by a simple linear transformation from a canonical c.W.p. in a separable Hilbert space. This representation is in terms of a series of independent real-valued Wiener processes and so has an interpretation as an approximation theorem. The weak stochastic integral is defined for the case the integrator is a c.W.p. in $B$ and the integrand is a stochastic linear operator from $B$ to a real separable Banach space $B_{1}$. The construction of the integral (Lemma 3.2), using the series representation of the c.W.p., is very easy and transparent; it results in a representation of the integral as a sum of scalar Ito integrals. However, the uniqueness of the construction is not evident and requires a somewhat tedious verification.* The cylindrical stochastic process determined by the weak integral with variable upper limit is readily seen to have weak sense analogs of the sample-path continuity and martingale properties of the Ito integral (Thm. 3.2).

The differential equation considered is formally

$$
\dot{X}_{t}=A X_{t}+\xi_{t}
$$

${ }^{*}$ We note the weak stochastic integral is also given an equivalent, coordinate-free definition in [4]. Furthermore, it is shown there that if the c.W.p. is in fact a $B$-valued Wiener process and if $B_{1}$ is a Hilbert space the integral agrees with that defined in [6] and determines a $B_{1}$-valued random variable. 
where $A$ does not depend on $t$ and $\xi_{t}$ is a stochastic forcing term. This is interpreted in integrated form (Eq. (4.1)) as an equation in a reflexive separable Banach space $B_{1}$ for a c.s.p. $X_{t}$. The operator $A$ is the infinitesimal generator of a class $C_{0}$ semigroup, and the integral of $\xi_{t}$ is a weak stochastic integral. The existence of an essentially unique c.s.p. $X_{t}$ that satisfies the equation and certain admissibility conditions is shown in Thm. 4.2. The proof of existence in Thm. 4.2 is a verification of the stochastic version of the variation-of-parameters formula using standard semigroup theory. An apparently nontrivial technical result that is needed and that may be of some interest is Thm. 4.1, which establishes the validity of an interchange of order of integration when one of the integrals is a weak stochastic integral.

In the special case that $B_{1}$ and $B$ are Hilbert spaces and the operator-valued function $\Phi$ is deterministic, Eq. 4.1 is the evolution equation treated in [1] in terms of weak processes, and is a special case of the equation studied in [3] referred to above. The approach we have taken is, however, rather closer to that of Curtain and Falb [6], which provided a background for this work despite the fact weak processes are not considered in [6].

\section{Preliminaries}

The following notations are used throughout the paper. The symbols $B$ and $H$, with or without subscripts refer to real separable Banach and Hilbert spaces, respectively. The adjoint space to $B$ is denoted $B^{*}$ as usual; $H$ and $H^{*}$ are identified. If $x \in B, y \in B^{*}$, the real number $y(x)$ is written $(x, y)_{B}$, or $(y, x)_{B^{*}}$ where $x$ is identified with an element of $B^{* *}$. In case the space is a Hilbert space $H,(x, y)_{H}$ is then the inner product. The domain and range of a linear transformation $A$ are denoted by $\mathscr{Q}(A)$ and $\mathscr{R}(A)$, respectively. The notation for the Banach space of bounded linear transformations from $B$ to $B_{1}$ is $L\left(B, B_{1}\right)$. Norms are often written with subscripts; the norm in $B$ is $\|\cdot\|_{B}$, and in $L\left(B, B_{1}\right)$ is $\|\cdot\|_{B B_{1}}$. The triple $(\Omega, \mathcal{Q}, m)$ is a probability space, with $\sigma$-algebra $\mathscr{Q}$ of subsets of $\Omega$ and probability measure $m$ on $\mathbb{Q}$. With $\mathbb{Q}$ given, $\left\{\mathbb{Q}_{t}\right\}_{t \geq 0}$ is a family of sub $\sigma$-algebras of $\mathbb{Q}$ with $\mathbb{Q}_{s} \subset \mathbb{Q}_{t}$ whenever $s \leq t$. It is assumed always that $\mathbb{Q}$ is complete and that all null sets of $\mathscr{Q}$ are contained in each $\mathbb{Q}_{t}$. If $\xi$ is a random variable on $(\Omega, Q, m)$, the expectation of $\xi, \int_{\Omega} \xi d m$, is often written $E \xi$.

Definition 2.1. A weak random variable (w.r.v.) $X$ in $B$ on $(\Omega, Q, m)$ is a linear map from $B^{*}$ into the linear space of real random variables (r.v.'s) on $(\Omega, Q, m)$. Thus $X y, y \in B^{*}$ is a r.v. A cylindrical stochastic process (c.s.p.) in $B$ on $(\Omega, \mathbb{Q}, m)$ is a family $\left\{X_{t}\right\}_{t \in T}$ of weak random variables on $(\Omega, \mathbb{Q}, m)$, with $T \subset R$.

Definition 2.2. A w.r.v. $X$ is uniformly square-integrable if there is $M>0$ such that

$$
E[X y]^{2}=\int_{\Omega}|X y|^{2} d m \leq M\|y\|_{B^{*}}^{2}
$$

for all $y \in B^{*}$. 
If $X$ is uniformly square-integrable it determines an element of $L\left(B^{*}, L_{2}\right)$, where $L_{2}$ is the Lebesgue space $L_{2}(\Omega, Q, m)$. This element of $L\left(B^{*}, L_{2}\right)$ is usually also denoted $X$.

The covariance operator is fundamental in what follows. We note first the following fact.

Lemma 2.1. Let $X$ be a uniformly square-integrable w.r.v. in $B$. Then there exists a unique bounded linear transformation $C$ in $L\left(B^{*}, B^{* *}\right)$ such that

$$
\left(y_{1}, C y_{2}\right)_{B^{*}}=\int_{\Omega}\left(X y_{1}\right)\left(X y_{2}\right) d m, \quad y_{1}, y_{2} \in B^{*} .
$$

Moreover $C$ is symmetric,

$$
\left(y_{1}, C y_{2}\right)_{B^{*}}=\left(y_{2}, C y_{1}\right)_{B^{*}} \quad \text { for all } y_{1}, y_{2} \in B^{*},
$$

and $C$ is positive,

$$
(y, C y)_{B^{*}} \geq 0 \quad \text { for all } y \in B^{*} .
$$

Proof. The integral $\int_{\Omega}\left(X y_{1}\right)\left(X y_{2}\right) d m$ defines a continuous bilinear functional. The existence of $C$ and the verification of its properties then follow in the usual way.

We are interested only in the case that $C$ is actually a map into $B$. Thus the definition of covariance operator to be used is restricted to this case.

Definition 2.3. Let $X$ be a uniformly square integrable w.r.v. If the operator $C$ given by Lemma 2.1 belongs to $L\left(B^{*}, B\right)$, it is said to be the covariance operator of $X$.

Thus, if $B$ is reflexive every uniformly square-integrable w.r.v. in $B$ has a covariance operator. Even if $B$ is not reflexive, there exist nontrivial w.r.v.'s which have covariance operators. In fact, every positive, symmetric $C \in L\left(B^{*}, B\right)$ is a covariance for some Gaussian w.r.v. $X$ on $B$ (see [2], Corollary 1.2); $X$ is then uniformly square-integrable.

A real Wiener process $\left\{\xi_{t}, \mathbb{Q}_{t}\right\}_{t \geq 0}$ is to be understood to be a real-valued Gaussian process with independent increments satisfying: (i) $\xi_{0}=0$ with probability one; (ii) $E \xi_{t}=0$; (iii) $E\left(\xi_{t}-\xi_{s}\right)^{2}=c|t-s|, c>0$; (iv) $\xi_{t}$ is measurable with respect to $\mathbb{Q}_{t}$; (v) $\left(\xi_{t}-\xi_{s}\right)$ is independent of $\mathcal{Q}_{s}$. We also refer simply to $\left\{\xi_{t}\right\}_{t \geq 0}$ as a Wiener process if all but conditions (iv) and (v) above are satisfied; of course $\mathbb{Q}_{t}$ can then be taken to be the $\sigma$-algebra generated by $\left\{\xi_{s}\right\}_{0 \leq s \leq t}$. All real Wiener processes that are introduced are assumed to be separable and hence continuous, i.e., to have continuous sample paths with probability one.

Definition 2.4. A c.s.p. $\left\{X_{t}\right\}_{t \leq T}$ has weakly independent increments if for any finite set of points $t_{0}<t_{1}<\cdots<t_{n}$ in $T$, and for any finite subset $\left\{y_{1}, \ldots, y_{n}\right\}$ of $B^{*}$ the random variables $\left(X_{t_{1}}-X_{t_{0}}\right) y_{1},\left(X_{t_{2}}-X_{t_{1}}\right) y_{2}, \ldots,\left(X_{t_{n}}-X_{t_{n-1}}\right) y_{n}$ are independent. 
Definition 2.5. A c.s.p. $\left\{W_{t}\right\}_{t \geq 0}$ in $B$ is a cylindrical Wiener process (c.W.p.) if (i) for any $y \in B^{*}\left\{W_{t} y\right\}_{t \geq 0}$ is a real Wiener process; (ii) $\left\{W_{t}\right\}_{t \geq 0}$ has weakly independent increments, and (iii) $W_{t}$ is uniformly square-integrable with covariance operator $t C$ where $C$ is a symmetric, positive operator in $L\left(B^{*}, B\right)$.

Let $\phi_{t}$ be the characteristic functional of $W_{t}$, given by

$$
\phi_{t}(y)=\int_{\Omega} e^{i\left[W_{t} y\right]} d m, \quad y \in B^{*} .
$$

Then in the definition just stated, with conditions (i) and (ii) unchanged, (iii) can be replaced by (iiia):

$$
\phi_{t}(y)=e^{-1 / 2 t(C y, y)_{B}}
$$

where $C$ is a symmetric, positive operator in $L\left(B^{*}, B\right)$.

The existence of a c.W.p. in any real separable Banach space $B$ is shown by the construction to follow. Actually the kind of example obtained is no more than a trivial transformation of a canonical c.W.p. in Hilbert space, and may be presumed known (see Remark 2.1. after Thm. 2.2). However Thm. 2.1 is included for completeness and because it suggests the representation given in Thm. 2.2. Let $\left\{W_{t}^{i}, t \geq 0\right\}_{i=1}^{\infty}$ be a sequence of continuous real Wiener processes defined on a common probability space $(\Omega, \mathscr{Q}, m)$ and with the following properties: (1) $E\left(W_{t}^{i}\right)^{2}=t$ for all $t \geq 0$ and for all $i=1,2, \ldots$; (2) $\left\{W_{t}^{i}, t \geq 0\right\}_{i=1}^{\infty}$ has independent components, i.e., for each finite set $\left\{i_{k}\right\}_{k=1}^{n}$ of distinct positive integers and for each finite set $\left\{t_{k}\right\}_{k=1}^{n}$ of nonnegative numbers the random variables $\left\{W_{t_{k}}^{i_{k}}\right\}_{k=1}^{n}$ are independent. Let $H$ be a real separable Hilbert space and let $A \in L(H, B) ; A$ is otherwise arbitrary. Let $\left\{e_{i}\right\}_{i=1}^{\infty}$ be a complete orthonormal system (c.o.n.s.) in $H$. For each $t \geq 0$ define a family $\left\{W_{t}^{n}\right\}_{n=1}^{\infty}$ of w.r.v.'s in $B$ by

$$
\left(\bar{W}_{t}^{n} y\right)(\omega)=\left(\sum_{i=1}^{n} W_{t}^{i}(\omega) A e_{i}, y\right)_{B}, \quad y \in B^{*}
$$

Theorem 2.1. The sequence $\left\{\bar{W}_{t}^{n} y\right\}_{n=1}^{\infty}$ converges in mean-square and a.s. $(m)$ for each $y \in B^{*}$. The limits define w.r.v.'s $W_{t}, t \geq 0$, with characteristic functionals

$$
\phi_{t}(y)=e^{-1 / 2 t\left(A A^{*} y, y\right)_{B}}, y \in B^{*}, \quad t \geq 0 .
$$

The c.s.p. $\left\{W_{t}\right\}_{t \geq 0}$ is a c.W.p.

Proof. The proof is only sketched. A standard calculation yields the mean-square convergence of the partial sums $\overline{W_{t}^{n}} y$, and a.s. convergence follows since these are sums of independent r.v.'s. A standard characteristic function argument shows that $\left\{W_{t}\right\}_{t \geq 0}$ has weakly independent Gaussian increments, and a similar calculation yields Eq. (2.2). All of this is somewhat similar to what follows in the proof of Thm. 2.2.

To show that the $W_{t} y$ are continuous one may argue as follows. The real processes $\left\{\bar{W}_{t}^{n} y\right\}_{t \geq 0}$ are Gaussian and have continuous sample paths a.s. $(m)$. It 
may be shown that for each $y \in B^{*}, \overline{W_{t}^{n}} y$ converges uniformly a.s. $(m)$ to $W_{t} y$ on any finite interval. The proof goes exactly as part of the proof of a well-known theorem of Ito and Nisio ([8], Thm. 5.2) after one has established the bound given below. Since

$$
\begin{aligned}
E\left|\bar{W}_{t}^{n} y-\bar{W}_{s}^{n} y\right|^{2} & =E\left|\sum_{i=1}^{n}\left(W_{t}^{i}-W_{s}^{i}\right)\left(A e_{i}, y\right)_{B}\right|^{2} \\
& =|t-s| \sum_{i=1}^{n}\left(e_{i}, A^{*} y\right)_{H}^{2} \leq|t-s|\left\|A^{*} y\right\|_{H}^{2}
\end{aligned}
$$

it follows that

$$
\begin{aligned}
E\left|\bar{W}_{t}^{n} y-\bar{W}_{s}^{n} y\right|^{4} & =3\left(E\left|\bar{W}_{t}^{n} y-\bar{W}_{s}^{n} y\right|^{2}\right)^{2} \\
& \leq 3|t-s|^{2} \text { constant }
\end{aligned}
$$

for all $n=1,2, \ldots$. The proof given in the reference cited applied here yields the conclusion that for each $y \in B^{*},\left(W_{t} y\right)(\omega)$ is a continuous function of $t$ except for $\omega$ 's in a set of $m$-measure zero (the exceptional set depending on $y$ ).

Any c.W.p. in $B$ can be represented by a limit of sums something like the one in the preceding theorem. To establish this representation we need the following decomposition theorem (see [2]).

Theorem (Baxendale). Let $C$ be a symmetric, positive transformation in $L\left(B^{*}, B\right)$. Then there is a separable Hilbert space $H$ and an injection $F$ in $L(H, B)$ such that $C=F F^{*}$.

With $F$ and $H$ as given by this theorem, we call $(F, H)$ a decomposition of $C$.

Theorem 2.2. Suppose $\left\{W_{t}\right\}_{t \geq 0}$ is a c.W.p. and that $t C$ is the covariance operator of $W_{t}, t \geq 0$. Let $(F, H)$ be a decomposition of $C$, and let $\left\{e_{i}\right\}_{i=1}^{\infty}$ be a c.o.n.s. for $\overline{\Re\left(F^{*}\right)}$ with the property that $\left\{\boldsymbol{e}_{i}\right\}_{i=1}^{\infty} \subset \Re\left(F^{*}\right)$. Then there exists a sequence $\left\{W_{t}^{i}, t \geq 0\right\}_{i=1}^{\infty}$ of independent real standard Wiener processes such that for each $y \in B^{*}, \sum_{i=1}^{n} W_{t}^{i}\left(F e_{i}, y\right)_{B} \rightarrow W_{t} y$ in mean-square and a.s. $(m)$.

Proof. Since $R\left(F^{*}\right)$ is a separable pre-Hilbert space a sequence $\left\{e_{i}\right\}_{i=1}^{\infty}$ with the required properties exists. Choose for $y_{i}, i=1,2, \ldots$, any element of $B^{*}$ such that $F^{*} y_{i}=e_{i}$. For each $t \geq 0, i=1,2, \ldots$, define the r.v. $W_{t}^{i}$ by $W_{t}^{i}=W_{t} y_{i}$. By the definition of a c.W.p. each $\left\{W_{t}^{i}, t \geq 0\right\}$ is a real Wiener process with

$$
E\left(W_{t}^{i}\right)^{2}=t\left(C y_{i}, y_{i}\right)=t\left\|F^{*} y_{i}\right\|_{H}^{2}=t
$$


For each $y \in B^{*}$,

$$
\begin{aligned}
E\left|\sum_{i=1}^{n} W_{t}^{i}\left(F e_{i}, y\right)_{B}-W_{t} y\right|^{2} & =E\left|W_{t}\left(\sum_{i=1}^{n} y_{i}\left(F e_{i}, y\right)_{B}-y\right)\right|^{2} \\
& =t\left\|A^{*}\left(\sum_{i=1}^{n} y_{i}\left(F e_{i}, y\right)_{B}-y\right)\right\|_{H}^{2} \\
& =t\left\|\sum_{i=1}^{n}\left(e_{i}, F^{*} y\right) e_{i}-y\right\|_{H}^{2} \rightarrow 0 \text { as } n \rightarrow \infty .
\end{aligned}
$$

Thus the partial sums converge in mean-square.

It remains to show the processes $W_{t}^{i}$ are independent. This will imply in particular that the r.v.'s $W_{t}^{i}, i=1,2, \ldots$, are independent so that the partial sums converge a.s. $(m)$. As a preliminary step we note the following. Let $s \leq t, i \neq j$, then

$$
\begin{aligned}
E\left[W_{s}^{j} W_{t}^{i}\right] & =E\left[W_{s}^{j}\left(W_{t}^{i}-W_{s}^{i}\right)\right]-E\left[W_{s}^{j} W_{s}^{i}\right] \\
& =E\left[\left(W_{s} y_{j}\right)\left(W_{t}-W_{s}\right)\left(y_{i}\right)\right]-E\left[W_{s} y_{j} W_{s} y_{i}\right]
\end{aligned}
$$

Since $\left\{W_{t}\right\}_{t \geq 0}$ has weakly independent increments the first of the terms above is zero. The second is also zero by the calculation,

$$
\begin{aligned}
E\left(W_{s} y_{j}\right)\left(W_{s} y_{i}\right) & =s\left(C y_{j}, y_{i}\right)_{B} \\
& =s\left(F F^{*} y_{j}, y_{i}\right)_{B} \\
& =s\left(e_{j}, e_{i}\right)_{H} \\
& =0 .
\end{aligned}
$$

Now consider a finite set of random variables $W_{t}^{i}$; for convenience take $i=1,2, \ldots, n$. The points $t_{i}$ are not necessarily distinct. Let $\phi\left(u_{1}, \ldots, u_{n}\right)$ be the characteristic function of the random vector $\left(W_{t_{1}}^{1}, \ldots, W_{t_{n}}^{n}\right)$. Then

$$
\begin{aligned}
\phi\left(u_{1}, \ldots, u_{n}\right) & =E\left[\exp \left(i \sum_{j=1}^{n} u_{j} W_{t_{j}}^{j}\right)\right] \\
& =\exp \left[-\frac{1}{2} E\left(\sum_{j=1}^{n} u_{j} W_{t_{j}}^{j}\right)^{2}\right] \\
& =\exp \left[-\frac{1}{2} \sum_{j=1}^{n} u_{j}^{2} E\left(W_{t_{j}}^{j}\right)^{2}\right] \\
& =\prod_{j=1}^{n} \exp \left[-\frac{1}{2} t_{j} u_{j}^{2}\right] .
\end{aligned}
$$


Remark 2.1. One consequence of Thm. 2.2 is that every c.W.p. in a separable Banach space $B$ can be expressed as a transformation of the canonical c.W.p. in a separable Hilbert space $H$ (a c.W.p. in $H$ with $\{t I\}_{t \geq 0}$ as the family of covariance operators) as follows. Let $\left\{W_{t}\right\}_{t \geq 0},\left\{e_{i}\right\}_{i=1}^{\infty}, C,(F, H)$ be as in Thm. 2.2. Define $W_{t}$ to be the canonical c.W.p. in $H$ given by

$$
\tilde{W}_{t} h=\sum_{i=1}^{\infty} W_{t}^{i}\left(e_{i}, h\right), \quad h \in H
$$

Let $F \tilde{W}_{t}$ be defined by $F \tilde{W}_{t} y=\tilde{W}_{t}\left(F^{*} y\right)$. Then the $W_{t}$ of Theorem 2.2 satisfies $W_{t} y=F \tilde{W}_{t} y$ a.s. $(m)$. Thus the Definition 2.5 can be replaced by the simpler definition: a c.W.p. $\left\{W_{t}\right\}_{t \geq 0}$ in $B$ is a process $W_{t}=F \tilde{W}_{t}$ a.s. $(m)$, where $F \in$ $L(H, B)$ and $\tilde{W}_{t}$ is a canonical c.W.p. in $H$ as given by Eq. (2.3). We note that formally $\tilde{W}_{t}$ is the integral of a "white noise" $\xi(t)$ in $H$, with $\operatorname{cov} \xi(t)=I$, as introduced by Bensoussan (see [3], p. 127).

We also note that in case the seminorm $|\cdot|$ on $H$ defined by $|h|=\|F h\|_{B}$ is a measurable seminorm in the sense of Gross [9], the c.W.p. $W_{t}$ reduces to a $B$-valued Wiener process that coincides with the $B$-valued Wiener process constructed by Gross via abstract Wiener space (see [4]).

One further fact about cylindrical Wiener processes is needed.

Lemma 2.2. Let $\left\{W_{t}\right\}_{t \geq 0}$ be a c.W.p. on $(\Omega, \mathbb{Q}, m)$. There exists a family $\left\{\mathbb{Q}_{t}\right\}_{t \geq 0}$ of sub $\sigma$-algebras of $\mathbb{Q}$ such that $\left\{W_{t} y, \mathbb{Q}_{t}\right\}_{t \geq 0}$ is a real Wiener process for each $y \in B^{*}$.

Proof. Let the covariance operator of $W_{t}$ be $t C$ and fix a decomposition $(F, H)$ of $C$. With $\left\{W_{t}^{i}, t \geq 0\right\}_{i=1}^{\infty}$ defined as in the preceding theorem, for each $t$ let $\mathbb{Q}_{t}^{i}=\sigma\left\{W_{s}^{i}, s \leq t\right\}$ (i.e., $\mathbb{Q}_{t}^{i}$ is the $\sigma$-algebra generated by $\left\{W_{s}^{i}, s \leq t\right\}$ ). For $0 \leq s<t$ let $\mathbb{Q}_{s t}^{i}=\sigma\left\{W_{t}^{i}-W_{s}^{i}\right\}$. Since the $\left\{W_{t}^{i}\right\}_{t \geq 0}$ are independent processes it follows that the family of $\sigma$-algebras $\left(\left\{\mathbb{Q}_{s t}^{i}\right\}_{i=1}^{\infty},\left\{\mathbb{Q}_{s}^{i}\right\}_{i=1}^{\infty}\right)$ is an independent family. Define $\tilde{\mathbb{Q}}_{s}$ to be $\sigma\left\{\mathbb{Q}_{s}^{i}, i=1,2, \ldots\right\}$ and $\mathscr{Q}_{s t}$ to be $\sigma\left\{\mathbb{Q}_{s t}^{i}, i=1,2, \ldots\right\}$. From a theorem on independence of compound $\sigma$-algebras (see [10], p. 237) it follows that $\tilde{\mathbb{Q}}_{s t}$ and $\tilde{\mathbb{Q}}_{s}$ are independent $\sigma$-algebras.

Let $N$ be the collection of $m$-null sets of $\mathscr{Q}$, and let $\mathcal{Q}_{s}=\sigma\left\{\tilde{\mathbb{Q}}_{s}, N\right\}$ and $\mathbb{Q}_{s t}=\sigma\left\{\tilde{\mathbb{Q}}_{s t}, N\right\}$. Since $\tilde{\mathbb{Q}}_{s}$ and $\tilde{\mathbb{Q}}_{s t}$ are independent, so are $\mathbb{Q}_{s}$ and $\mathbb{Q}_{s t}$. For each $y \in B^{*}$ and for each $n=1,2, \ldots,\left(\overline{W_{t}^{n}}-\overline{W_{s}^{n}}\right)(y)$ as defined in the preceding theorem is $\mathbb{Q}_{s t}$-measurable and hence independent of $\mathbb{Q}_{s}$. We show that $\left(W_{t}-\right.$ $\left.W_{s}\right)(y)$ is $\mathbb{Q}_{s t}$-measurable and hence independent of $\mathbb{Q}_{s}$. In fact, since $\left(\vec{W}_{t}^{n}-\right.$ $\left.\overrightarrow{W_{s}^{n}}\right)(y) \rightarrow\left(W_{t}-W_{s}\right)(y)$ a.s. $(m)$ as $n \rightarrow \infty$, it follows that $\left(\overline{W_{t}^{n}}-\overline{W_{s}^{n}}\right)(y) \rightarrow\left(W_{t}-\right.$ $\left.W_{s}\right)(y)$ a.s. $\left(m_{s t}\right)$, where $m_{s t}$ is the restriction of $m$ to $\mathbb{Q}_{s t}$. Thus the limit $\left(W_{t}-W_{s}\right)(y)$ is $\mathbb{Q}_{s t}$-measurable.

The same argument may be applied to yield the conclusion that $W_{t} y$ is $\mathbb{Q}_{t}$-measurable for all $t \geq 0$. Thus $\left\{W_{t} y, \mathbb{Q}_{t}\right\}_{t \geq 0}$ is a real-valued Wiener process for each $y \in B^{*}$. 


\section{Weak Stochastic Integrals}

In this section the weak stochastic integral $(w) \int_{0}^{t} \Phi(s) d W_{s}$ is defined as a c.s.p. in $B_{1}$ for $0 \leq t \leq t_{1}$. The integrator is a c.W.p. $\left(W_{s}, Q_{s}\right)$ in $B$ and the integrand is a stochastic operator-valued function belonging to the class $M\left(B, B_{1}\right)$, to be defined.

Definition 3.1. Let $(\Omega, \mathcal{Q}, m)$ be given, and $\left\{Q_{t}\right\}_{t \geq 0}$ be a nondecreasing family of sub $\sigma$-algebras of $Q$. The class $M\left(B, B_{1}\right)$ is the class of functions $\Phi:\left[0, t_{1}\right] \times \Omega \rightarrow$ $L\left(B, B_{1}\right)$ that satisfy the following conditions:

(1) For each $y \in B_{1}^{*}, \Phi^{*} y$ is strongly measurable on $\mathscr{B}\left[0, t_{1}\right] \times \mathbb{Q}$, and for each $t \in\left[0, t_{1}\right], \Phi^{*}(t) y$ is $\mathbb{Q}_{t}$-strongly measurable.

$\left[0, t_{1}\right]$.

(2) $\int_{\left[0, t_{1}\right] \times \Omega}\|\Phi(s, \omega)\|_{B, B_{1}}^{2} d(l \times m)<\infty$, where $l$ is the Lebesgue measure on

Remark 3.1. Because of the assumed separability of $B$ and $B_{1}$ the strong measurability of $\Phi^{*} y$ for each $y \in B_{1}^{*}$ implies first the strong measurability of $\Phi z$ with respect to $\mathscr{B}\left[0, t_{1}\right] \times Q$ for each $z \in B$ and then the measurability of $\|\Phi(\cdot, \cdot)\|$. Thus the integral in condition (2) is always defined. The first assertion follows from the observation that $(\Phi z, y)_{B_{1}}=\left(\Phi^{*} y, z\right)_{B^{*}}$ is measurable, a fortiori for all $z \in B$ and all $y \in B_{1}^{*}$. The second requires a short conventional argument using the measurability of $\Phi z$ and the separability of $B$.

As already indicated, the weak stochastic integral is defined here by a construction involving a series of scalar Ito integrals. In particular, let $(F, H)$ be a decomposition of $C$ (where $t C: B^{*} \rightarrow B$ is the covariance operator for $W_{t}$ ); let $\left\{e_{i}\right\}_{i=1}^{\infty}$ be an o.n. basis for $\overline{\Re\left(F^{*}\right)}, e_{i} \in \Re\left(F^{*}\right)$; let $y_{i} \in B^{*}$ be such that $F^{*} y_{i}=e_{i}$, and let $W_{t}^{i}=W_{t} y_{i}$. All this is as described in the previous section. We consider the Ito integrals

$$
\int_{0}^{t}\left(\Phi(s) F e_{i}, y\right)_{B_{1}} d W_{s}^{i}, \quad 0 \leq t \leq t_{1}, \quad i=1,2, \ldots,
$$

where $y \in B_{1}^{*}, \Phi \in M\left(B, B_{1}\right)$. Clearly, the integrand satisfies the measurability conditions required for an Ito integral and is square-integrable $l \times m$. Using the standard theory for Ito integrals one may readily verify the following lemma.

Lemma 3.1. For $i \neq j, 0 \leq t \leq t_{1}$,

$$
E \int_{0}^{t}\left(\Phi(s) F e_{i}, y\right)_{B_{1}} d W_{s}^{i} \int_{0}^{t}\left(\Phi(u) F e_{j}, y\right)_{B_{1}} d W_{s}^{j}=0 .
$$

Lemma 3.2. With $\int_{0}^{t}\left(\Phi(s) F e_{i}, y\right)_{B_{1}} d W_{s}^{i}$ as specified for the previous lemma, the series $\sum_{i=1}^{\infty} \int_{0}^{t}\left(\Phi(s) F e_{i}, y\right)_{B_{1}} d W_{s}^{i}, 0 \leq t \leq t_{1}$, converges in mean-square on $(\Omega, Q, m)$ for each $y \in B_{1}^{*}$. 
Proof.

$$
\begin{aligned}
E\left|\sum_{i=m}^{n} \int_{0}^{t}\left(\Phi(s) F e_{i}, y\right)_{B_{1}} d W_{s}^{i}\right|^{2} \\
\quad=\sum_{i=m}^{n} \int_{0}^{t} E\left(\Phi(s) F e_{i}, y\right)_{B_{1}}^{2} d s \\
\quad=\int_{0}^{t} E \sum_{i=m}^{n}\left(\left(e_{i}, F^{*} \Phi^{*}(s) y\right) e_{i}, F^{*} \Phi^{*}(s) y\right)_{H} d s
\end{aligned}
$$

where the first equality follows from Lemma 3.1 and a property of Ito integrals. Let $P_{m}$ be the orthogonal projection in $H$ on the span of $\left\{e_{1}, \ldots, e_{m}\right\}$. Then the above integral can be written

$$
\begin{aligned}
\int_{0}^{t} E & \left(\left(P_{n}-P_{m}\right) F^{*} \Phi^{*}(s) y, F^{*} \Phi^{*}(s) y\right)_{H} d s \\
\quad & \int_{0}^{t} E\left\|\left(P_{n}-P_{m}\right) F^{*} \Phi^{*}(s) y\right\|_{H}^{2} d s \\
& \leq \int_{0}^{t} E\left\|\left(I-P_{m}\right) F^{*} \Phi^{*}(s) y\right\|_{H}^{2} d s, n \geq m .
\end{aligned}
$$

Since $\left\|\left(I-P_{m}\right) F^{*} \Phi^{*}(s) y\right\|_{H} \leq\left\|F^{*}\right\| \cdot\|y\| \cdot\|\Phi(s)\|$, which is square-integrable $l \times m$, and since $\left\|\left(I-P_{m}\right) F^{*} \Phi^{*}(s) y\right\| \rightarrow 0$ as $m \rightarrow \infty$, the integrals approach zero as $m \rightarrow \infty, n>m$, by dominated convergence. The assertion follows.

For convenience we denote the mean-square limit given by this lemma by $I_{t}(\Phi, y)$. The above construction makes it appear that $I_{t}(\Phi, y)$ depends on the particular decomposition $(F, H)$ used, and on the choice of $\left\{e_{i}\right\}_{i=1}^{\infty}$ and $\left\{y_{i}\right\}_{i=1}^{\infty}$. We show below that in fact it does not. The proof given depends on the use of operator-valued stochastic step functions, which have not yet been required. A function $\tilde{\Phi} \in M\left(B, B_{1}\right)$ with the property that for some set of points $0=s_{1}<s_{2}<$ $\cdots<s_{k+1}=t_{1}$

$$
\tilde{\Phi}(s, \omega)=\tilde{\Phi}\left(s_{k}, \omega\right), \quad s_{k} \leq s<s_{k+1}
$$

is called simply a stochastic step function. Clearly $\tilde{\boldsymbol{\Phi}}^{*}$ is then a step function in $s$ and $\int_{0}^{t_{1}} E\left\|\tilde{\Phi}^{*}(s)\right\|_{B_{1}^{*} B^{*}}^{2} d s<\infty$

Theorem 3.1. Given any $\Phi \in M\left(B, B_{1}\right)$, there exists a sequence $\left\{\Phi_{n}\right\}_{n=1}^{\infty}$ of stochastic step functions such that for any $y \in B_{1}^{*}$

$$
\lim _{n \rightarrow \infty} \int_{0}^{t_{1}} E\left\|\Phi^{*}(s) y-\Phi_{n}^{*}(s) y\right\|_{B^{*}}^{2} d s=0
$$

The proof follows very closely a standard proof of the scalar version of this theorem and is not given (see [4] for a similar theorem). 
Lemma 3.3. Let $\Phi \in M\left(B, B_{1}\right)$ and let $\left\{\Phi_{n}\right\}_{n=1}^{\infty}$ be a sequence of stochastic step functions as given by Theorem 3.1. Let $I_{t}(\Phi, y), 0 \leq t \leq t_{1}$, be formed with any $(F, H),\left\{e_{i}\right\}_{i=1}^{\infty}$ and $\left\{y_{i}\right\}_{i=1}^{\infty}$ satisfying the required conditions. Then for any $y \in B_{1}^{*}$, $I_{t}\left(\Phi_{n}, y\right) \rightarrow I_{t}(\Phi, y)$ in $L_{2}(\Omega, \mathbb{Q}, m)$ as $n \rightarrow \infty$.

Proof. Put $\Theta_{n}(s)=\Phi(s)-\Phi_{n}(s)$. Then $\Theta_{n} \in M\left(B, B_{1}\right)$ and

$$
\lim _{n \rightarrow \infty} \int_{0}^{t} E\left\|\Theta_{n}^{*}(s) y\right\|_{B^{*}}^{2} d s=0 \text { for all } y \in B_{1}^{*} .
$$

For any positive integer $N$,

$$
\begin{aligned}
E\left|\sum_{i=1}^{N} \int_{0}^{t}\left(\Phi(s) F e_{i}, y\right) B_{1} d W_{s}^{i}-\sum_{i=1}^{N} \int_{0}^{t}\left(\Phi_{n}(s) F e_{i}, y\right)_{B_{1}} d W_{s}^{i}\right|^{2} \\
\quad=E\left|\sum_{i=1}^{N} \int_{0}^{t}\left(\Theta_{n}(s) F e_{i}, y\right)_{B_{1}} d W_{s}^{i}\right|^{2} \\
\quad=\sum_{i=1}^{N}\left|\int_{0}^{t}\left(\Theta_{n}(s) F e_{i}, y\right)_{B_{1}} d W_{s}^{i}\right|^{2} \quad \text { (by Lemma 3.1) } \\
\quad=\sum_{i=1}^{N} \int_{0}^{t} E\left(e_{i}, F^{*} \Theta_{n}^{*}(s) y\right)_{H}^{2} d s . \\
=\int_{0}^{t} E\left[\sum_{i=1}^{N}\left(e_{i}, F^{*} \Theta_{n}^{*}(s) y\right)_{H}^{2}\right] d s \\
\quad \leq \int_{0}^{t} E\left\|F^{*} \Theta_{n}^{*}(s) y\right\|_{H}^{2} d s \leq\left\|F^{*}\right\| \int_{0}^{t} E\left\|\Theta_{n}^{*}(s) y\right\|_{B_{1}^{*}}^{2} d s .
\end{aligned}
$$

Given $\varepsilon>0$, choose $n_{0}$ such that the value of the dominating expression in the above inequality is less that $(\varepsilon / 3)^{2}$ for $n \geq n_{0}$. Then

$$
\begin{aligned}
& \left\|I_{t}(\Phi, y)-I_{t}\left(\Phi_{n}, y\right)\right\|_{L_{2}} \\
& \leq\left\|I_{i}(\Phi, y)-\sum_{i=1}^{N} \int_{0}^{t}\left(\Phi(s) F e_{i}, y\right)_{B_{1}} d W_{s}^{i}\right\|_{L_{2}} \\
& \quad+\frac{\varepsilon}{3}+\left\|I_{t}\left(\Phi_{n}, y\right)-\sum_{i=1}^{N} \int_{0}^{t}\left(\Phi_{n}(s) F e_{i}, y\right)_{B_{1}} d W_{s}^{i}\right\|_{L_{2}}
\end{aligned}
$$

for $n \geq n_{0}$. With $n$ temporarily fixed, one can take $N$ sufficiently large that the first and third terms on the right side of the above inequality are less than $\varepsilon / 3$. Thus

$$
\left\|I_{t}(\Phi, y)-I_{t}\left(\Phi_{n}, y\right)\right\|_{L_{2}} \leq \varepsilon
$$

for all $n \geq n_{0}$. 
Lemma 3.4. $I_{t}(\Phi, y)$ does not depend on the decomposition $(F, H)$ of $C$, nor on the sequences $\left\{e_{i}\right\}_{i=1}^{\infty},\left\{y_{i}\right\}_{i=1}^{\infty}$ used in its definition.

Proof. Consider two decompositions of $C,(F, H)$ and $(\bar{F}, \bar{H})$, and corresponding sequences $\left\{e_{i}\right\}_{i=1}^{\infty},\left\{y_{i}\right\}_{i=1}^{\infty}$ and $\left\{\bar{e}_{i}\right\}_{i=1}^{\infty},\left\{\bar{y}_{i}\right\}_{i=1}^{\infty}$. Let $I_{t}(\Phi, y)$ denote the meansquare limit of $\sum_{i=1}^{N} \int_{0}^{t}\left(\Phi(s) F e_{i}, y\right)_{B_{1}} d W_{s}^{i}$ and $\tilde{I}_{r}(\Phi, y)$ denote the mean-square limit of $\sum_{i=1}^{N} \int_{0}^{t}\left(\Phi(s) \bar{F} \bar{e}_{i}, y\right)_{B_{1}} d \bar{W}_{s}^{i}$, where $\bar{W}_{s}^{i}=W_{s} \bar{y}_{i}$. By the previous lemma, it is sufficient to prove $I_{t}(\Phi, y)=\bar{I}_{t}(\Phi, y)$ when $\Phi$ is a stochastic step function.

We consider first the case that $\Phi$ consists of a single step, e.g.,

$$
\begin{aligned}
\Phi(u, \omega) & =\Phi_{1}(\omega), & & 0 \leq u<s \\
& =0, & & s \leq u
\end{aligned}
$$

where $\Phi_{1}(\omega) \in L\left(B, B_{1}\right), \Phi_{1}^{*} y$ is $\mathbb{Q}_{0}$-measurable for each $y \in B_{1}^{*}$ and $E\left\|\Phi_{1}\right\|^{2}=$ $E\left\|\Phi_{1}^{*}\right\|^{2}<\infty$. For this case $I_{t}(\Phi, y)\left(\bar{I}_{t}(\Phi, y)\right)$ is the mean-square limit of

$$
Z_{n}=\sum_{i=1}^{n}\left(\Phi_{1} F e_{i}, y\right) W_{s}^{i}\left(\bar{Z}_{m}=\sum_{j=1}^{m}\left(\Phi_{1} \bar{F} \bar{e}_{j}, y\right) \bar{W}_{s}^{j}\right) .
$$

To start with we show that

$$
\lim _{m \rightarrow \infty} \lim _{n \rightarrow \infty}\left\|Z_{n}-\bar{Z}_{m}\right\|_{L_{2}}=0 \text {. }
$$

In fact, using Lemma 3.1 and the definition of $W_{s}^{i}$,

$$
\begin{aligned}
\left\|Z_{n}-\bar{Z}_{m}\right\|_{L_{2}}^{2}= & E\left|\sum_{i=1}^{n}\left(e_{i}, F^{*} \Phi_{1}^{*} y\right) W_{s}^{i}-\sum_{j=1}^{m}\left(\bar{e}_{i}, \bar{F}^{*} \Phi_{1}^{*} y\right) \bar{W}_{s}^{j}\right|^{2} \\
= & s \sum_{i=1}^{n} E\left(e_{i}, F^{*} \Phi_{1}^{*} y\right)^{2}+s \sum_{j=1}^{m} E\left(\bar{e}_{j}, \bar{F}^{*} \Phi_{1}^{*} y\right)^{2} \\
& -2 E \sum_{i=1}^{n} \sum_{j=1}^{m}\left(W_{s} y_{i}\right)\left(W_{s} \bar{y}_{j}\right)\left(e_{i}, F^{*} \Phi_{1}^{*} y\right)\left(\bar{e}_{j}, \bar{F}^{*} \bar{\Phi}_{1}^{*} y\right) .
\end{aligned}
$$

The third term on the right side of Eq. (3.1) is equal to

$$
\begin{aligned}
-2 s & \sum_{i=1}^{n} \sum_{j=1}^{m}\left(C y_{i}, \bar{y}_{j}\right) E\left(e_{i}, F^{*} \Phi_{1}^{*} y\right)\left(\bar{e}_{j}, \bar{F}^{*} \bar{\Phi}_{1}^{*} y\right) \\
& =-2 s E \sum_{i=1}^{n} \sum_{j=1}^{m}\left(F^{*} y_{i}, F^{*} \bar{y}_{j}\right)\left(e_{i}, F^{*} \Phi_{1}^{*} y\right)\left(\bar{e}_{j}, \bar{F}^{*} \Phi_{1}^{*} y\right) \\
& =-2 s E\left[\sum_{j=1}^{m}\left(\bar{e}_{j}, \bar{F}^{*} \Phi_{1}^{*} y\right) \sum_{i=1}^{n}\left(e_{i}, F^{*} \bar{y}_{j}\right)\left(e_{i}, F^{*} \Phi_{1}^{*} y\right)\right] .
\end{aligned}
$$


For each $m$, the bracketed expression in Eq. (3.2) is dominated for all $n$ by a function integrable on $(\Omega, Q, m)$. In fact, by the Schwarz inequality, its square is less than or equal to

$$
\begin{aligned}
& \sum_{j=1}^{m}\left(\bar{e}_{j}, \bar{F}^{*} \Phi_{1}^{*} y\right)^{2} \cdot \sum_{j=1}^{m}\left[\sum_{i=1}^{n}\left(e_{i}, F^{*} \bar{y}_{j}\right)\left(e_{i}, F^{*} \Phi_{1}^{*} y\right)\right]^{2} \\
& \quad \leq\left\|\bar{F}^{*} \Phi_{1}^{*} y\right\|^{2} \cdot \sum_{j=1}^{m}\left[\sum_{i=1}^{n}\left(e_{i}, F^{*} \bar{y}_{j}\right)^{2} \sum_{i=1}^{n}\left(e_{i}, F^{*} \Phi_{1}^{*} y\right)^{2}\right] \\
& \quad \leq\left\|\bar{F}^{*} \Phi_{1}^{*} y\right\|^{2} \cdot \sum_{j=1}^{m}\left\|F^{*} \bar{y}_{j}\right\|^{2} \cdot\left\|F^{*} \Phi_{1}^{*} y\right\|^{2} \\
& \quad=\left\|\bar{F}^{*} \Phi_{1}^{*} y\right\|^{2} \cdot\left\|F^{*} \Phi_{1}^{*} y\right\|^{2} \cdot \sum_{j=1}^{m}\left(\bar{e}_{j}, \bar{e}_{j}\right) \\
& \quad \leq m\left\|F^{*}\right\|^{2} \cdot\left\|F^{*}\right\|^{2} \cdot\left\|\Phi_{1}^{*} y\right\|^{4} .
\end{aligned}
$$

Since $E\left\|\Phi_{1}^{*} y\right\|^{2}<\infty$, the limit on $n$ may be interchanged with the expectation for the bracketed expression in Eq. (3.2) to yield

$$
\begin{aligned}
\lim _{n \rightarrow \infty} E[\ldots] & =E\left[\sum_{j=1}^{m}\left(\bar{e}_{j}, \bar{F}^{*} \Phi_{1}^{*} y\right) \cdot \lim _{n \rightarrow \infty} \sum_{i=1}^{n}\left(e_{i}, F^{*} \bar{y}_{j}\right)\left(e_{i}, F^{*} \Phi_{1}^{*} y\right)\right] \\
& =E\left[\sum_{j=1}^{m}\left(\bar{e}_{j}, \bar{F}^{*} \Phi_{1}^{*} y\right)\left(F^{*} \bar{y}_{j}, F^{*} \Phi_{1}^{*} y\right)\right] \\
& =E \sum_{j=1}^{m}\left(\bar{e}_{j}, \bar{F}^{*} \Phi_{1}^{*} y\right)\left(\bar{F}^{*} \bar{y}_{j}, \bar{F}^{*} \Phi_{1}^{*} y\right) \\
& =E \sum_{j=1}^{m}\left(\bar{e}_{j}, \bar{F}^{*} \Phi_{1}^{*} y\right)\left(\bar{e}_{j}, \bar{F}^{*} \Phi_{1}^{*} y\right) \\
& =E \sum_{j=1}^{m}\left(\bar{e}_{j}, \bar{F}^{*} \Phi_{1}^{*} y\right)^{2}
\end{aligned}
$$

where the identity $\left(\bar{F}^{*} z, \bar{F}^{*} w\right)=(C z, w)=\left(F^{*} z, F^{*} w\right)$ has been used. Thus, from Eq. (3.2) and the calculation above

$$
\lim _{n \rightarrow \infty}\left\|Z_{n}-\bar{Z}_{m}\right\|_{L_{2}}^{2}=-s E \sum_{j=1}^{m}\left(\bar{e}_{j}, \bar{F}^{*} \Phi_{1}^{*} y\right)^{2}+s E\left\|F^{*} \Phi_{1}^{*} y\right\|_{L_{2}}^{2} .
$$

Now,

$$
\begin{gathered}
\left\|I_{t}(\Phi, y)-\tilde{I}_{t}(\Phi, y)\right\|_{L_{2}}^{2} \leq 3\left\{\left\|I_{t}(\Phi, y)-Z_{n}\right\|_{L_{2}}^{2}+\left\|Z_{n}-\bar{Z}_{m}\right\|_{L_{2}}^{2}\right. \\
\left.+\left\|\bar{Z}_{m}-\bar{I}_{t}(\Phi, y)\right\|_{L_{2}}^{2}\right\}
\end{gathered}
$$


for all $n, m$. Hence, taking the limit on $n$ with $m$ fixed and using Eq. (3.3),

$$
\begin{aligned}
\left\|I_{t}(\Phi, y)-\bar{I}_{t}(\Phi, y)\right\|_{L_{2}}^{2} \leq 3\{ & \left\|Z_{m}-I_{t}(\Phi, y)\right\|_{L_{2}}^{2} \\
& \left.+s E\left\|F^{*} \Phi_{1}^{*} y\right\|_{L_{2}}^{2}-s E \sum_{j=1}^{m}\left(e_{i}, F^{*} \Phi_{1}^{*} y\right)^{2}\right\}
\end{aligned}
$$

for all $m$. Taking the limit on $m$ gives $I_{t}(\Phi, y)=\bar{I}_{t}(\Phi, y)$ for the special $\Phi$ considered.

If now one considers arbitrary stochastic step functions the verification is an elaboration of that just given with the only new feature being the existence of "cross-terms" in the expansion of $\left\|Z_{n}-Z_{m}\right\|_{L_{2}}^{2}$. By a standard and easy calculation, depending ultimately on the fact $E\left\{W_{t} y_{j}-W_{s} y_{j} \mid \mathbb{Q}_{s}\right\}=0, s<t$, it may be seen these terms are zero.

The weak stochastic integral, denoted $(w) \int_{0}^{t} \Phi(s) d W_{s}, 0 \leq t \leq t_{1}$, can now be defined by

$$
\left((w) \int_{0}^{t} \Phi(s) d W_{s}\right)(y)=I_{t}(\Phi, y), \quad y \in B_{1}^{*} .
$$

Theorem 3.2. Let $\Phi$ belong to the class $M\left(B, B_{1}\right)$, defined with respect to the

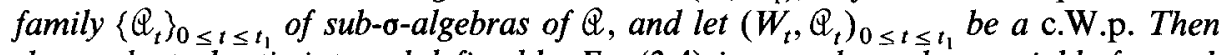
the weak stochastic integral defined by Eq. (3.4) is a weak random variable for each $t$ and $a$ c.s.p. for $0 \leq t \leq t_{1}$. Furthermore:

(1) $\left((w) \int_{0}^{t} \Phi(s) d W_{s}\right)(y)$ has a modification with continuous sample paths for $m$-almost all $\omega \in \Omega$.

(2) $\left((w) \int_{0}^{t} \Phi(s) d W_{s}\right)(y)$ is a martingale for each $y \in B^{*}$.

(3) $E\left((w) \int_{0}^{t} \Phi(u) d W_{u}\right)(x) \cdot\left((w) \int_{0}^{s} \Phi(u) d W_{u}\right)(y)$ $=\int_{0}^{\min (s, t)} E\left(\Phi(u) C \Phi^{*}(u) x, y\right)_{B_{1}} d u, \quad x, y \in B_{1}^{*}$,

and hence the covariance $D \in L_{1}\left(B_{1}^{*}, B_{1}\right)$ of $(w) \int_{0}^{t} \Phi(s) d W_{s}$ exists and is given by

$$
D y=\int_{0}^{t} E\left[\Phi(s) C \Phi^{*}(s) y\right] d s, \quad y \in B_{1}^{*} \text {. }
$$

Proof. By Lemmas 3.2 and 3.4 the weak stochastic integral is well defined for each $t$ as a mapping from $B_{1}^{*}$ into the space of real-valued random variables on $(\Omega, Q, m)$. It is clearly linear, so for fixed $t$ it is a weak random variable. 
To prove the assertion (2) we note first that since $\int_{0}^{t}\left(\Phi(s) F e_{i}, y\right)_{B_{1}} d W_{s}^{i}$ is a martingale with respect to the family $\left\{\mathbb{Q}_{t}\right\}_{t \geq 0}$, so also is

$$
X_{t}^{n} \triangleq \sum_{i=1}^{n} \int_{0}^{t}\left(\Phi(s) F e_{i}, y\right)_{B_{1}} d W_{s}^{i}
$$

Further, $X_{t} \triangleq I_{t}(\Phi, y)$ is $Q_{t}$-measurable. Then to show that $X_{t}$ is a martingale with respect to $\left\{Q_{t}\right\}_{t \geq 0}$, one needs only to show that for each $s \leq t$

$$
\int_{\Lambda} X_{t} y d m=\int_{\Lambda} X_{s} y d m, \quad \Lambda \in \mathbb{Q}_{s}
$$

But since

$$
\begin{aligned}
\left|\int_{\Lambda} X_{t} y d m-\int_{\Lambda} X_{t}^{n} y d m\right|^{2} & \leq\left|\int_{\Lambda}\right| X_{t} y-\left.X_{t}^{n} y\right|^{2} d m \\
& \leq E\left|X_{t} y-X_{t}^{n} y\right|^{2} \rightarrow 0 \text { as } n \rightarrow \infty
\end{aligned}
$$

by Lemma 3.2 , and since $\int_{\Lambda}\left(X_{t}^{n} y-X_{s}^{n} y\right) d m=0$, Eq. (3.5) follows from the triangle inequality.

The assertion (1) is proved by using the facts: (i) both $X_{t}^{n}$ and $X_{t}$ as just defined are martingales; (ii) $X_{t}^{n}$ converges in mean-square to $X_{t}$; (iii) each $X_{t}^{n}$ has a modification that has continuous sample paths for $m$-almost all $\omega \in \Omega$. Then one need only follow the usual proof for the scalar case, using a standard martingale inequality and the Borel-Cantelli lemma (see [8], p. 446, for example). The proof referred to starts with approximation by stochastic step functions, which is different from what we have here, but the arguments are the same.

The assertion (3) is proved by the following calculation. Let $s \leq t$ :

$$
\begin{aligned}
& E\left((w) \int_{0}^{t} \Phi(u) d W_{u}\right)(x) \cdot\left((w) \int_{0}^{s} \Phi(u) d W_{u}\right)(y) \\
& =\lim _{n \rightarrow \infty} E\left(\sum_{i=1}^{n} \int_{0}^{t}\left(\Phi(u) F e_{i}, x\right)_{B_{1}} d W_{u}^{i} \cdot \sum_{j=1}^{n} \int_{0}^{s}\left(\Phi(v) F e_{j}, y\right) d W_{v}^{j}\right.
\end{aligned}
$$

by Lemma 3.2 and continuity of the inner products. By Lemma 3.1 and Ito integral theory, this last expression is readily shown to be equal to

$$
\int_{0}^{s} E\left(\Phi(u) C \Phi^{*}(u) x, y\right)_{H} d u
$$




\section{Stochastic Linear Differential Equations}

We consider stochastic differential equations (always written here in the integrated form) of the following type

$$
X_{t} y=X y+\int_{0}^{t} X_{s}\left(A^{*} y\right) d s+\left((w) \int_{0}^{t} \Phi(s, \omega) d W_{s}\right)(y)
$$

where $X$ is an initial weak r.v. in a reflexive Banach space $B_{1}$ and $A$ is the infinitesimal generator of a class $C_{0}$ semigroup $\{T(t)\}_{t>0}$ on $B_{1}$.

The proof of the existence theorem we obtain requires some further facts about integrals. In particular it is necessary to discuss iterated integrals involving both weak stochastic integration and Bochner or Lebesgue integration. These may be written

$$
\begin{aligned}
& X_{1}=(w) \int_{0}^{t_{1}}\left[\int_{0}^{t_{1}} \Psi(t, s, \omega) d s\right] d W_{t}, \\
& X_{2}=\int_{0}^{t_{1}}\left[(w) \int_{0}^{t_{1}} \Psi(t, s) d W_{t}\right] d s .
\end{aligned}
$$

These expressions are purely formal at this point and need to be properly defined. This is accomplished below in Lemmas 4.1 and 4.2.

The following definition is an analogue of Def. 3.1.

Definition 4.1. Let $(\Omega, \mathbb{Q}, m)$ be given, and $\left\{\mathscr{Q}_{t}\right\}_{t \geq 0}$ be a nondecreasing family of sub $\sigma$-algebras of $Q$. The class $N\left(B, B_{1}\right)$ is the class of functions $\Psi(t, s, \omega)$, $\Psi:\left[0, t_{1}\right] \times\left[0, t_{1}\right] \times \Omega \rightarrow L\left(B, B_{1}\right)$, that satisfy the following conditions:

(1) For each $y \in B_{1}^{*}, \Psi^{*} y$ is strongly measurable on $\mathscr{B}\left[0, t_{1}\right] \times \mathscr{B}\left[0, t_{1}\right] \times \mathbb{Q}$, and for each $t \in\left[0, t_{1}\right], \Psi^{*}(t) y$ is $\mathscr{B}\left[0, t_{1}\right] \times Q_{t}$ strongly measurable.

(2) $\int_{\left[0, t_{1}\right] \times\left[0, t_{1}\right] \times \Omega}\|\Psi(t, s, \omega)\|_{B B_{1}}^{2} d(l \times l \times m)<\infty$.

We note, as in Remark 3.1. following Def. 3.1, that $\Psi z, z \in B$, is strongly measurable with respect to $\Re\left[0, t_{1}\right] \times \Re\left[0, t_{1}\right] \times Q$ and $\|\Psi\|$ is measurable.

Let $\Psi \in N\left(B, B_{1}\right)$. Then $\Psi(t, \cdot, \omega)$ as an $L\left(B, B_{1}\right)$-valued function of $s$ is not necessarily strongly measurable, so the integral $\int_{0}^{t_{1}} \Psi(t, s, \omega) d s$ may not be defined in the sense of Bochner. However $\Psi(t, \cdot, \omega) x, x \in B$, is Bochner integrable. Thus we can define $\int_{0}^{t_{1}} \Psi(t, s, \omega) d s$ to be the operator $\Phi(t, \omega) \in L\left(B, B_{1}\right)$ given by

$$
\Phi(t, \omega) x=\int_{0}^{t_{1}} \Psi(t, s, \omega) x d s
$$


Lemma 4.1. If $\Psi \in N\left(B, B_{1}\right)$ then $\Phi$ as defined in Eq. (4.4) belongs to the class $M\left(B, B_{1}\right)$. The iterated integral $X_{1}$ is well-defined as a weak stochastic integral by

$$
X_{1} y=\text { m.s. } \lim _{n \rightarrow \infty} \sum_{i=1}^{n} \int_{0}^{t_{1}}\left(\int_{0}^{t_{1}} \Psi(t, s) F e_{i} d s, y\right)_{B_{1}} d W_{t}^{i}, \quad y \in B_{1}^{*} .
$$

Proof. Since $\Psi$ satisfies the measurability conditions of Def. 4.1 it is clear that $\Phi$ satisfies the measurability conditions of Def. 3.1. Further, $\|\Phi(s, \omega)\|$ $\leq \int_{0}^{t_{1}}\|\Psi(t, s, \omega)\| d s$, from which it follows from the Schwarz inequality and Fubini theorem that

$$
\int_{\left[0, t_{1}\right] \times \Omega}\|\Phi(s, \omega)\|^{2} d(l \times m) \leq t_{1}^{2} \int_{\left[0, t_{1}\right] \times\left[0, t_{1}\right] \times \Omega}\|\Psi(t, s, \omega)\|^{2} d(l \times l \times m) .
$$

Since $\Phi(t) F e_{i}=\int_{0}^{t_{1}} \Psi(t, s) F e_{i} d s$, the second assertion is immediate.

Lemma 4.2. If $\Psi \in N\left(B, B_{1}\right)$ then for each $y \in B_{1}^{*},\left((w) \int_{0}^{t_{1}} \Psi(t, s) d W_{t}\right)(y)$ determines a stochastic process with parameter $s$ which has a measurable modification with sample functions integrable on $\left[0, t_{1}\right], m-a . s$. The iterated integral $X_{2}$ is a weak random variable defined by

$$
X_{2} y=\int_{0}^{t_{1}}\left[(w) \int_{0}^{t_{1}} \Psi(t, s) d W_{t}\right](y) d s .
$$

Proof. From the Fubini theorem it follows that for a.e. $s, \Psi(\cdot, s, \cdot) \in M\left(B, B_{1}\right)$. Thus $Y_{s} \triangleq(w) \int_{0}^{t_{1}} \Psi(t, s) d W_{t}$ is well defined for a.e. $s$. Using an idea taken from [9], we now construct a c.s.p. $\tilde{Y}_{s}$ such that for each $y \in B_{1}^{*}, Y_{s} y=\tilde{Y}_{s} y$ a.e. $(l \times m)$. For convenience $t_{1}$ is set equal to 1 in what follows.

Consider the probability space $(\tilde{\Omega}, \tilde{\mathbb{Q}}, \tilde{m})$ with $\tilde{\Omega}=[0,1] \times \Omega, \tilde{\mathcal{Q}}=\mathscr{B}[0,1] \times \mathscr{Q}$, $\tilde{m}=l \times m$. Let $\tilde{\mathbb{Q}}_{t} \triangleq \mathscr{B}[0,1] \times \mathbb{Q}_{t}$; obviously $\left\{\tilde{\mathbb{Q}}_{t}\right\}_{t \geq 0}$ is a nondecreasing family of sub $\sigma$-algebras of $\tilde{\mathcal{Q}}$. For each $t \in[0,1]$ and $\tilde{\omega}=(s, \omega) \in \tilde{\Omega}$ define the function $\tilde{\boldsymbol{\Phi}}$ : $[0,1] \times \Omega \rightarrow L\left(B, B_{1}\right)$ by $\Phi(t, \tilde{\omega})=\Psi(t, s, \omega)$. It follows that $\tilde{\Phi} \in \tilde{M}\left(B, B_{1}\right)$, where $\tilde{M}\left(B, B_{1}\right)$ is a class as given in Def. 3.1 , but with respect to $(\tilde{\Omega}, \tilde{\mathbb{Q}}, \tilde{m})$ and $\left\{\tilde{\mathbb{E}}_{t}\right\}_{t \geq 0}$.

A c.W.p. $\left\{\tilde{W}_{t}, \tilde{Q}_{t}\right\}$ can be constructed as follows. With $(F, H),\left\{e_{i}\right\}_{i=1}^{\infty}$ and $W_{t}^{i}$ as in Lemma 3.1, put $\tilde{W}_{t}^{i}(\tilde{\omega}) \triangleq W_{t}^{i}(\omega), \tilde{\omega}=(s, \omega) \in \tilde{\Omega}, i=1,2, \ldots$. Then $\sum_{i=1}^{n} \tilde{W}_{t}^{i} F e_{i}$ is a $B$-valued random variable. It may be shown for each $y \in B^{*}$ that $\lim _{n \rightarrow \infty}\left(\sum_{i=1}^{n} \tilde{W}_{t}^{i} F e_{i}, y\right)_{B}$ exists a.e. $(m)$ and defines a weak random variable $\tilde{W}_{t}$; further, $\left\{\tilde{W}_{t}, \tilde{\mathscr{Q}}_{t}\right\}$ is a c.W.p. (see Thm. 2.1). Thus the weak stochastic integral (w) $\int_{0}^{1} \tilde{\Phi}(t, \tilde{\omega}) d \tilde{W}_{t}$ is well defined, and $\left((w) \int_{0}^{1} \tilde{\Phi}(t, \tilde{\omega}) d \tilde{W}_{t}\right)(y), y \in B_{1}^{*}$, is $\tilde{Q}-$ measurable; i.e., it is $\mathscr{G}[0,1] \times \mathbb{Q}$-measurable. 
Now take a sequence $\left\{\tilde{\Phi}_{n}(t, \tilde{\omega})\right\}_{n=1}^{\infty}$ of stochastic step functions belonging to $\tilde{M}\left(B, B_{1}\right)$ that approximate $\tilde{\Phi}$ in the sense of Thm. 3.1. Put $\Phi_{n}(t, s, \omega) \triangleq \tilde{\Phi}_{n}(t, \tilde{\omega})$, $\tilde{\omega}=(s, \omega)$. By the Fubini theorem we have for $y \in B_{1}^{*}$

$$
\begin{aligned}
& \int_{0}^{1}\left[\int_{0}^{1} E\left\|\Phi_{n}^{*}(t, s) y-\Psi^{*}(t, s) y\right\|_{B^{*}}^{2} d s\right] d t \\
&=\int_{0}^{1} \tilde{E}\left\|\tilde{\Phi}_{n}^{*}(t) y-\tilde{\Phi}^{*}(t) y\right\|_{B^{*}}^{2} d t \rightarrow 0 \text { as } n \rightarrow \infty .
\end{aligned}
$$

Hence for almost all $s \in[0,1]$, for some subsequence (still denoted $\Phi_{n}$ ) it follows that

$$
\int_{0}^{1} E\left\|\Phi_{n}^{*}(t, s) y-\Psi^{*}(t, s) y\right\|_{B^{*}}^{2} d t \rightarrow 0
$$

Then by Lemma 3.3, for almost all $s \in[0,1]$ and all $y \in B_{1}^{*}$

$$
\left((w) \int_{0}^{1} \Psi(t, s) d W_{t}\right)(y)=\lim _{n \rightarrow \infty}\left((w) \int_{0}^{1} \Phi_{n}(t, s) d W_{t}\right)(y)
$$

where the limit is a strong limit in $L_{2}(\Omega, Q, m)$. It is not yet clear that $\left((w) \int_{0}^{1} \Psi(t, s) d W_{t}\right)(y)$ is $\mathscr{B}[0,1] \times \mathbb{Q}$-measurable. However we show below that it is equal a.e. $(l \times m)$ to $\left((w) \int_{0}^{1} \tilde{\Phi}(t) d \tilde{W}_{t}\right)(y)$, which is $\mathscr{B}[0,1] \times \mathscr{Q}$-measurable. Once this is done the lemma is proved, because by $\mathrm{Thm}$. 3.2 this last integral is square-integral $(\tilde{m}=l \times m)$ and hence determines a real-valued stochastic process for which almost all sample functions are Lebesgue integrable.

Since $E\left[\left((w) \int_{0}^{1} \tilde{\Phi}(t, \tilde{\omega}) d \tilde{W}_{t}\right)(y)\right]^{2}$ exists for a.e. $s$, as do also the corresponding integrals with $\tilde{\Phi}_{n}$, one can write for a.e. $s$ and $y \in B_{1}^{*}$

$$
\begin{aligned}
& \left\|\left((w) \int_{0}^{1} \tilde{\Phi}(t, \tilde{\omega}) d \tilde{W}_{t}\right)(y)-\left((w) \int_{0}^{1} \Psi(t, s, \omega) d W_{t}\right)(y)\right\|_{L_{2}(\Omega, \mathcal{Q}, m)} \\
& \quad \leq F_{n}+G_{n}+H_{n},
\end{aligned}
$$

where

$$
\begin{aligned}
F_{n} & =\left\|\left((w) \int_{0}^{1} \tilde{\Phi}(t, \tilde{\omega}) d \tilde{W}_{t}\right)(y)-\left((w) \int_{0}^{1} \tilde{\Phi}_{n}(t, \tilde{\omega}) d \tilde{W}_{t}\right)(y)\right\|_{L_{2}(\Omega, \mathbb{Q}, m)} \\
G_{n} & =\left\|\left((w) \int_{0}^{1} \tilde{\Phi}_{n}(t, \tilde{\omega}) d \tilde{W}_{t}\right)(y)-\left((w) \int_{0}^{1} \Phi_{n}(t, s, \omega) d W_{t}\right)(y)\right\|_{L_{2}(\Omega, \mathbb{Q}, m)} \\
H_{n} & =\left\|\left((w) \int_{0}^{1} \Phi_{n}(t, s, \omega) d W_{t}\right)(y)-\left((w) \int_{0}^{1} \Psi(t, s, \omega) d W_{t}\right)(y)\right\|_{L_{2}(\Omega, \mathbb{Q}, m)} .
\end{aligned}
$$

Straightforward calculations show that $G_{n}=0$ for almost all $s \in[0,1]$ and all 
$n=1,2, \ldots$. By Lemma 3.3, $\int_{0}^{1} F_{n}^{2} d s \rightarrow 0$ as $n \rightarrow \infty$; so some subsequence of the $F_{n}$ 's approaches zero for almost all $s \in[0,1]$. Finally, for a.e. $s$, each $\Phi_{n}(\cdot, s, \cdot) \in$ $M\left(B, B_{1}\right)$ and is an approximating sequence for $\Psi(\cdot, s, \cdot)$ in the sense of Thm. 3.1, and $H_{n} \rightarrow 0$ as $n \rightarrow \infty$ for a.e. $s \in[0,1]$. Thus the assertion concerning equality of the two stochastic integrals is proved.

Theorem 4.1. With $X_{1} y$ and $X_{2} y$ as defined in Lemmas 4.1 and 4.2, $X_{1} y=X_{2} y$ a.e. $(m)$ for each $y \in B_{1}^{*}$. Thus the weak random variables $X_{1}$ and $X_{2}$ are equal.

Proof. We show that $X_{1}=X_{2}$ for the case $\Psi(t, s, \omega)$ is a stochastic step function in the variable $t$. By identifying $\Psi(t, s, \omega)$ with $\tilde{\Phi}(t, \tilde{\omega})$ as in the preceding lemma it is straightforward to carry through the passage to the limit for each of $X_{1}$ and $X_{2}$ to extend the result to arbitrary $\Psi \in N\left(B, B_{1}\right)$.

Let $\Psi \in N\left(B, B_{1}\right)$ be of the form

$$
\Psi(t, s, \omega)=\sum_{i=1}^{n} \Phi^{i}(s, \omega) 1_{\left[t_{i}, t_{i+1}\right]}(t)
$$

where $0=t_{1}<t_{2}<\cdots<t_{n+1}=1$ (again take $t_{1}=1$ ). Then, for each positive integer $k$ and all $y \in B_{1}^{*}$

$$
\begin{aligned}
\left\|X_{1} y-X_{2} y\right\|_{L_{2}(\Omega, \mathbb{Q}, m)} \leq & \left\|X_{1} y-P_{k}\right\|_{L_{2}(\Omega, \mathbb{Q}, m)} \\
& +\left\|X_{2} y-P_{k}\right\|_{L_{2}(\Omega, \mathbb{Q}, m)}
\end{aligned}
$$

where

$$
P_{k}(\omega)=\int_{0}^{1} Q_{k}(s, \omega) d s
$$

with

$$
Q_{k}(s, \omega)=\sum_{j=1}^{k} \sum_{i=1}^{n}\left(W_{t_{i+1}}^{j}-W_{t_{i}}^{j}\right)\left(\Phi^{i}(s, \omega) F e_{j}, y\right)_{B_{1}} .
$$

By the definition of $X_{1}$, and $\Psi$ as given by Eq. (4.8) and the standard calculation used in Lemma 3.2,

$$
\left\|X_{1} y-P_{k}\right\|_{L_{2}(\Omega, Q, m)}=\int_{0}^{1}\left[\int_{0}^{1} E \sum_{j=k+1}^{\infty}\left(\Psi(t, s) F e_{j}, y\right)_{B_{1}}^{2} d t\right] d s .
$$

The sum inside the integral approaches zero a.e. $(l \times l \times m)$ as $k \rightarrow \infty$ and is dominated for all $k$ by $\|C\|_{B^{*} B}\|y\|^{2}\|\Psi(t, s)\|^{2}$. Since $\int_{0}^{1} \int_{0}^{1} E\|\Psi(t, s)\|^{2} d t d s<\infty$, the dominated convergence theorem applies and $\left\|X_{1} y-P_{k}\right\|_{L_{2}(\Omega, \mathbb{Q}, m)} \rightarrow 0$ as $k \rightarrow \infty$. 
Now

$$
\begin{aligned}
\left\|X_{2} y-P_{k}\right\|_{L_{2}(\Omega, Q, m)}^{2} & =E\left|X_{2} y-P_{k}\right|^{2} \\
& =E\left|\int_{0}^{1}\left[\left((w) \int_{0}^{1} \Psi(t, s, \omega) d W_{t}\right)(y)-Q_{k}(s, \omega)\right] d t\right|^{2} \\
& \leq E \int_{0}^{1}\left[\left((w) \int_{0}^{1} \Psi(t, s, \omega) d W_{t}\right)(y)-Q_{k}(s, \omega)\right]^{2} d s
\end{aligned}
$$

But, using the development of Lemma 4.2 , one may put $\tilde{Q}_{k}(\tilde{\omega})=Q_{k}(s, \omega)$, $\tilde{\omega}=(s, \omega) \in \tilde{\Omega}$. Then

$$
\begin{aligned}
\tilde{Q}_{k}(\tilde{\omega}) & =\sum_{j=1}^{k} \sum_{i=1}^{n}\left(\tilde{W}_{t_{i+1}}^{j}(\tilde{\omega})-\tilde{W}_{t_{i}}^{j}(\tilde{\omega})\right)\left(\tilde{\Phi}^{i}(\tilde{\omega}) F e_{j}, y\right)_{B_{1}} \\
& =\sum_{j=1}^{n}(w) \int_{0}^{1}\left(\tilde{\Phi}(t, \tilde{\omega}) F e_{j}, y\right)_{B} d \tilde{W}_{t}^{j}
\end{aligned}
$$

Since $\left((w) \int_{0}^{1} \Psi(t, s, \omega) d W_{t}\right)(y)=\left((w) \int_{0}^{1} \tilde{\Phi}(t, \tilde{\omega}) d \tilde{W}_{t}\right)(y)$ the right-hand side of the above inequality may be rewritten so that we have

$$
\left\|X_{2} y-P_{k}\right\|_{L_{2}(\Omega, \mathcal{Q}, m)}^{2} \leq \tilde{E}\left|\left((w) \int_{0}^{1} \tilde{\Phi}(t, \tilde{\omega}) d \tilde{W}_{t}\right)(y)-\tilde{Q}_{k}(\tilde{\omega})\right|^{2}
$$

This expression approaches zero as $k \rightarrow \infty$ by the definition of the stochastic integral.

One more preliminary fact is needed, an adaptation of the theorem of Hille on interchange of a closed linear transformation with a Bochner integral.

Lemma 4.3. Let $X$ be a uniformly square-integrable weak random variable and $y(\cdot)$ a $B^{*}$-valued function on $\left[0, t_{1}\right]$ continuous in the strong topology of $B^{*}$. Then $\int_{0}^{t_{1}}(X y(s))(\omega) d s$ is well-defined as a Lebesgue integral for $m$-almost all $\omega \in \Omega$. Moreover,

$$
\int_{0}^{t_{1}}(X y(s))(\omega) d s=X\left[\int_{0}^{t_{1}} y(s) d s\right](\omega) \quad \text { a.e. }(m)
$$

where the integral on the right is a Bochner integral.

Proof. The uniform square-integrability of $X$ and the strong continuity of $y$ imply the real-valued stochastic process $\{X y(s)\}_{s \in\left[0, t_{1}\right]}$ is continuous in $m$-measure and hence has a separable, measurable version (which we still denote by $\left.\{X y(s)\}_{s \in\left[0, t_{1}\right]}\right)$. Let $D$ be the transformation in $L\left(B^{*}, L_{2}(\Omega, Q, m)\right)$ that is 
determined by $X$. Then

$$
\begin{aligned}
\left(\int_{0}^{t_{1}} E|X y(s)| d s\right)^{2} & \leq t_{1} \int_{0}^{t_{1}} E|X y(s)|^{2} d s \\
& =t_{1} \int_{0}^{t_{1}}\|D y(s)\|_{L_{2}}^{2} d s \leq t_{1}\|D\|_{B^{*} L_{2}}^{2} \int_{0}^{t_{1}}\|y(s)\|_{B^{*}}^{2} d s<\infty .
\end{aligned}
$$

Thus by the Fubini theorem $\int_{0}^{t_{1}} X y(s) d s$ exists for $m$-almost all $\omega \in \Omega$ and is $m$-integrable.

Since

$$
\left(D \int_{0}^{t_{1}} y(s) d s\right)(\omega)=\left(X \int_{0}^{t_{1}} y(s) d s\right)(\omega) \quad \text { a.e. }(m)
$$

it follows by the theorem of Hille referred to that the proof of the lemma will be complete once it is verified that

$$
\left(\int_{0}^{t_{1}} D y(s) d s\right)(\omega)=\int_{0}^{t_{1}}(X y(s))(\omega) d s \quad \text { a.e. }(m) .
$$

Let $\left\{y_{n}(\cdot)\right\}_{n=1}^{\infty}$ be a sequence of simple functions in $B^{*}$ that converges uniformly on $\left[0, t_{1}\right]$ to $y(s)$. It is clear that Eq. (4.9) holds if $y$ is replaced by $y_{n}, n=1,2, \ldots$. By definition of the Bochner integral, $\int_{0}^{t_{1}} D y_{n}(s) d s \rightarrow \int_{0}^{t_{1}} D y(s) d s$ in $L_{2}(\Omega, \mathbb{Q}, m)$. To establish the convergence of the sequence of integrals to the right side of Eq. (4.9) for $m$-almost all $\omega$, we have:

$$
E\left|\int_{0}^{t_{1}}(X y(s))(\omega) d s-\int_{0}^{t_{1}}\left(X y_{n}(s)\right)(\omega) d s\right|^{2} \leq t_{1} \int_{0}^{t_{1}} E\left|X\left(y(s)-y_{n}(s)\right)\right|^{2} d s .
$$

Now,

$$
E\left|X\left(y(s)-y_{n}(s)\right)(\omega)\right|^{2} \leq\|D\|_{B^{*} L_{2}}^{2}\left\|y_{n}(s)-y(s)\right\|_{B^{*}}^{2} \rightarrow 0
$$

uniformly on $\left[0, t_{1}\right]$ as $n \rightarrow \infty$. Thus, by dominated convergence $\int_{0}^{t_{1}} E \mid X(y(s)-$ $\left.y_{n}(s)\right)\left.(\omega)\right|^{2} d s \rightarrow 0$ as $n \rightarrow \infty$.

We now return to a consideration of Eq. (4.1). First the conditions that are to hold are summarized, and then the class of solutions to be considered is defined. The space $B_{1}$ is a real, separable, reflexive Banach space. Let

(i) $\{T(t)\}_{t \geq 0}$ be a class $C_{0}$ semigroup defined on $B_{1}$ with infinitesimal generator $A$;

(ii) $\left\{W_{t}, \mathbb{Q}_{t}\right\}_{t \geq 0}$ be a cylindrical Wiener process in a real Banach space $B$, defined on a probability space $(\Omega, \mathbb{Q}, m)$, with covariance operator $C \in L\left(B^{*}, B\right)$

(iii) $\Phi(s, \omega)$ be defined on $\left[0, t_{1}\right] \times \Omega$, and $\Phi(\cdot, \cdot) \in M\left(B, B_{1}\right)$ with respect to $(\Omega, Q, m)$ and the family of sub $\sigma$-algebras $\left\{Q_{t}\right\}_{t \geq 0}$; 
(iv) $X$ be a uniformly square-integrable weak random variable in $B_{1}$ with respect to $(\Omega, Q, m)$ with the further property that the real random variables $\left\{X y, y \in B_{1}^{*}\right\}$ are all $Q_{0}$-measurable.

Since $B_{1}$ is reflexive, $\left\{T^{*}(t)\right\}_{t \geq 0}$ is a class $C_{0}$ semigroup on $B_{1}^{*}$ with infinitesimal generator $A^{*}$ (see [5], p. 52). Denote by $\mathscr{D}(A)$, $\mathcal{D}\left(A^{*}\right)$ the respective domains in $B_{1}, B_{1}^{*}$ of $A, A^{*}$; each is dense in its respective space.

Definition 4.2. A c.s.p. $\left\{X_{t}\right\}_{t \in\left[0, t_{1}\right]}$ in $B_{1}$ is said to be an admissible solution to Eq. (4.1) if for each $y \in B_{1}^{*}$ :

(a) $\left\{X_{t} y\right\}_{t \in\left[0, t_{1}\right]}$ is continuous in mean-square;

(b) $\left\{X_{t} y\right\}_{t \in\left[0, t_{1}\right]}$ is nonanticipative relative to $\left\{\mathbb{Q}_{t}\right\}_{t \geq 0}$;

(c) for each $t \in\left[0, t_{1}\right], X_{t}$ is uniformly square-integrable; and if for each $y \in$ D $\left(A^{*}\right):$

(d) $\left\{X_{t} y\right\}_{t \in\left[0, t_{1}\right]}$ satisfies Eq. (4.1) a.s. $(m)$;

(e) $\left\{X_{t} y\right\}_{t \in\left[0, t_{1}\right]}$ has continuous sample paths a.s. $(m)$.

Equation (4.1) is said to have a unique admissible solution if it has an admissible solution $\left\{X_{t}\right\}_{t \in\left[0, t_{1}\right]}$, and if for any other admissible solution $\left\{Z_{t}\right\}_{t \in\left[0, t_{1}\right]}$ it is true that for each $y \in B_{1}^{*}$

$$
m\left\{\sup _{t \in\left[0, t_{1}\right]}\left|X_{t} y-Z_{t} y\right|>0\right\}=0 .
$$

Remark 4.1. If $\left\{X_{t}\right\}_{t \in\left[0, t_{1}\right\}}$ is an admissible solution to Eq. (4.1) then $\left\{X_{t} y\right\}_{t \in\left[0, t_{1}\right]}$ has a separable, measurable version for each $y \in B_{1}^{*}$. We always assume such a version is chosen. Then the set $\left\{\sup _{t \in\left[0, t_{1}\right]}\left|X_{t} y-Z_{t} y\right|>0\right\}$ is measurable.

Theorem 4.2. Let the conditions (i), (ii), (iii), (iv) listed above be satisfied. Then the stochastic differential equation (4.1) has a unique admissible solution $\left\{X_{t}\right\}_{t \in\left[0, t_{1}\right]}$ such that $X_{0}=X$ (in the sense that for each $y \in B_{1}^{*}, X_{0} y=X y$ a.e. $(m)$ ). Furthermore, a particular solution is given by the c.s.p.

$$
X_{t}=T(t) X+(w) \int_{0}^{t} T(t-s) \Phi(s) d W_{s}
$$

where the c.s.p. $T(t) X$ is defined by

$$
(T(t) X)(y)=X\left(T^{*}(t) y\right)
$$

Proof. (Existence). We first show that $X_{t}$ as given by Eq. (4.10) is mean-square continuous (i.e. $X_{t} y$ is mean-square continuous). The process $T(t) X$ is meansquare continuous. In fact, if $t>0$ and $D \in L\left(B_{1}^{*}, L_{2}(\Omega, \mathbb{Q}, m)\right)$ is the operator determined by $X$,

$$
\begin{aligned}
& E|(T(t+s) X)(y)-(T(t) X)(y)|^{2} \\
& \quad=E\left|X T^{*}(t+s) y-X T^{*}(t) y\right|^{2} \\
& \quad \leq\|D\|_{B_{1}^{*} L_{2}}^{2}\left\|T^{*}(t)\right\|_{B_{1} B_{1}^{*}}^{2}\left\|T^{*}(s) y-y\right\|_{B_{1}^{*}}^{2} \rightarrow 0
\end{aligned}
$$

as $s \rightarrow 0$. 
Consider the stochastic integral in Eq. (4.10). Define

$$
\Psi(t, s, \omega)= \begin{cases}T(t-s) \Phi(s, \omega), & s \leq t \\ 0, & s>t\end{cases}
$$

It is easy to check that $\Psi \in N_{1}\left(B, B_{1}\right)$, so that the integral in question is well-defined. With $\Delta>0, y \in B_{1}^{*}$,

$$
\begin{aligned}
((w) & \left.\int_{0}^{t+\Delta} T(t+\Delta-s) \Phi(s) d W_{s}\right)(y)-\left((w) \int_{0}^{t} T(t-s) \Phi(s) d W_{s}\right)(y) \\
= & \left((w) \int_{0}^{t}(T(\Delta)-I) T(t-s) \Phi(s) d W_{s}\right)(y) \\
& +\left((w) \int_{t}^{t+\Delta} T(t-s+\Delta) \Phi(s) d W_{s}\right)(y)
\end{aligned}
$$

The first integral on the right side of Eq. (4.12) can be written

$$
\left((w) \int_{0}^{t} T(t-s) \Phi(s) d W_{s}\right)\left(\left(T^{*}(\Delta)-I^{*}\right)(y)\right),
$$

as follows immediately from the definition of the integral $\left(I^{*}\right.$ denotes the identity on $\left.B_{1}^{*}\right)$. By Thm. 3.2 and the strong continuity of the semigroup $\left\{T^{*}(t)\right\}$,

$$
\begin{aligned}
& E\left|\left((w) \int_{0}^{t}(T(\Delta)-I) T(t-s) \Phi(s) d W_{s}\right)(y)\right|^{2} \\
& \quad=\int_{0}^{t} E\left\|F^{*} \Phi^{*}(s) T^{*}(t-s)\left(T^{*}(\Delta)-I^{*}\right) y\right\|_{H}^{2} d s \\
& \quad \leq\|F\|_{H B}^{2} M\left\|\left(T^{*}(\Delta)-I^{*}\right) y\right\|_{B^{*}}^{2} \int_{0}^{t} E\|\Phi(s)\|_{B B_{1}}^{2} d s
\end{aligned}
$$

where $M$ is such that $\|T(s)\|_{B_{1} B_{1}} \leq M$ for all $s \in[0, t]$.

For the second integral on the right side of Eq. (4.12) one has

$$
\begin{aligned}
& E\left|\left((w) \int_{t}^{t+\Delta} T(t-s+\Delta) \Phi(s) d W_{s}\right)(y)\right|^{2} \\
& \quad=\int_{t}^{t+\Delta} E\left\|F^{*} \Phi^{*}(s) T^{*}(t-s+\Delta)(y)\right\|_{H}^{2} d s,
\end{aligned}
$$

which obviously approaches zero as $\Delta \rightarrow 0$. Hence $\left((w) \int_{0}^{t} T(t-s) \Phi(s) d W_{s}\right)(y)$ is mean-square continuous.

For fixed $t$ and with $\Psi$ as defined above in Eq. (4.11) the integral $\left((w) \int_{0}^{r} \Psi(t, s) d W_{s}\right)(y)$ is $\mathscr{Q}_{r}$-measurable; hence, in particular, when $r=t$, the 
integral $\left((w) \int_{0}^{t} T(t-s) \Phi(s) d W_{s}\right)(y)$ is $\mathbb{Q}_{t}$-measurable. This fact together with the hypotheses on the $\mathbb{Q}_{0}$-measurability of the initial weak random variable shows that the $X_{t}$ of Eq. (4.10) satisfies condition (b) in Definition 4.2. The uniform square-integrability of this $X_{t}$ follows immediately from Thm. 3.2 and the requirement on $X$.

We now show that for $\left.y \in G_{1}\right)\left(A^{*}\right), X_{t} y$ as given by Eq. (4.10) satisfies Eq. (4.1). Since the function $\Psi$ defined in Eq. (4.11) belongs to $N\left(B, B_{1}\right)$, Thm. 4.1 applies to the iterated integrals with integrand $\Psi$. Thus for $y \in \mathscr{D}\left(A^{*}\right)$ one may write

$$
\begin{aligned}
& \int_{0}^{t}\left[(w) \int_{0}^{s} T(s-\sigma) \Phi(\sigma) d W_{\sigma}\right]\left(A^{*} y\right) d s \\
& \quad=\left((w) \int_{0}^{t}\left[\int_{\sigma}^{t} T(s-\sigma) \Phi(\sigma) d s\right] d W_{\sigma}\right)\left(A^{*} y\right) \quad \text { a.e. }(m) .
\end{aligned}
$$

By definition of the weak stochastic integral,

$$
\begin{aligned}
& \left((w) \int_{0}^{t}\left[\int_{\sigma}^{t} T(s-\sigma) \Phi(\sigma) d s\right] d W_{\mathrm{o}}\right)\left(A^{*} y\right) \\
& =\sum_{i=1}^{\infty} \int_{0}^{t}\left(\left(\int_{\sigma}^{t} T(s-\sigma) \Phi(\sigma) d s\right) F e_{i}, A^{*} y\right)_{B_{1}} d W_{\sigma}^{i}
\end{aligned}
$$

where the limit is understood to be in mean-square $(m)$ and the inner integral on the right is the operator as defined in Eq. (4.4). Now, for all $i=1,2, \ldots$, and for $m$-almost all $\omega$,

$$
\begin{aligned}
& \left(\left(\int_{\sigma}^{t} T(s-\sigma) \Phi(\sigma) d s\right) F e_{i}, A^{*} y\right)_{B_{1}} \\
& \quad=\left(\int_{\sigma}^{t} T(s-\sigma) \Phi(\sigma) F e_{i} d s, A^{*} y\right)_{B_{1}} \\
& \quad=\int_{\sigma}^{t}\left(T(s-\sigma) \Phi(\sigma) F e_{i}, A^{*} y\right)_{B_{1}} d s \\
& \quad=\int_{\sigma}^{t}\left(\Phi(\sigma) F e_{i}, T^{*}(s-\sigma) A^{*} y\right)_{B_{1}} d s \\
& \quad=\left(\Phi(\sigma) F e_{i}, \int_{\sigma}^{t} T^{*}(s-\sigma) A^{*} y d s\right)_{B_{1}} \\
& =\left(\Phi(\sigma) F e_{i}, T^{*}(t-\sigma) y\right)_{B_{1}}-\left(\Phi(\sigma) F e_{i}, y\right)_{B_{1}} .
\end{aligned}
$$


Thus,

$$
\begin{aligned}
\int_{0}^{t}[(w) & \left.\int_{0}^{s} T(s-\sigma) \Phi(\sigma) d W_{\sigma}\right]\left(A^{*} y\right) d s \\
= & \sum_{i=1}^{\infty} \int_{0}^{t}\left(\Phi(\sigma) F e_{i}, T^{*}(t-\sigma) y\right)_{B_{1}} d W_{\sigma}^{i} \\
& -\sum_{i=1}^{\infty} \int_{0}^{t}\left(\Phi(\sigma) F e_{i}, y\right)_{B_{1}} d W_{\sigma}^{i} \\
= & \left((w) \int_{0}^{t} T(t-\sigma) \Phi(\sigma) d W_{\sigma}\right)(y) \\
& -\left((w) \int_{0}^{t} \Phi(\sigma) d W_{\sigma}\right)(y) .
\end{aligned}
$$

Since the initial weak random variable is uniformly square-integrable, Lemma 4.3 applies to give:

$$
\begin{aligned}
\left(X T^{*}(t) y\right)(\omega) & =X\left[\int_{0}^{t} T^{*}(s) A^{*} y d s+y\right](\omega) \\
& =\int_{0}^{t}\left(X T^{*}(s) A^{*} y\right)(\omega) d s-X y(\omega)
\end{aligned}
$$

for $m$-almost all $\omega \in \Omega$. Note that the last equality shows that the real-valued process $\left\{X T^{*}(t) y\right\}_{t \in\left\{0, t_{1}\right]}$ has continuous sample paths with probability one.

With $X_{s}$ as given by Eq. (4.10), and with substitutions from Eqs. (4.13) and (4.14), one has

$$
\begin{aligned}
\int_{0}^{t} X_{s} A^{*} y d s= & \int_{0}^{t} X T^{*}(s) A^{*} y d s \\
& +\int_{0}^{t}\left[(w) \int_{0}^{s} T(s-\sigma) \Phi(\sigma) d W_{\sigma}\right]\left(A^{*} y\right) d s \\
= & X T^{*}(t) y-X y+\left((w) \int_{0}^{t} T(t-\sigma) \Phi(\sigma) d W_{\sigma}\right)(y) \\
& -\left((w) \int_{0}^{t} \Phi(\sigma) d W_{\sigma}\right)(y) \quad \text { a.e. }(m) .
\end{aligned}
$$

A rearrangement of terms in the above equation yields

$$
\begin{aligned}
X_{t}(y) & =X T^{*}(t) y+\left((w) \int_{0}^{t} T(t-s) \Phi(s) d W_{s}\right)(y) \\
& =X y+\int_{0}^{t} X_{s} A^{*} y d s+\left((w) \int_{0}^{t} \Phi(s) d W_{s}\right)(y) \quad \text { a.e. }(m) .
\end{aligned}
$$

Thus the differential equation is satisfied for all $y \in \mathscr{Q}\left(A^{*}\right)$. Since each term on 
the right-hand side of this last equation has continuous sample paths a.s. $(m)$, so also has the solution $X_{t}(y)$.

(Uniqueness). This proof follows exactly the scheme of a uniqueness proof for a nonstochastic equation (see [1]). Let $\left\{X_{t}\right\},\left\{Z_{t}\right\}, t \in\left[0, t_{1}\right]$ be two admissible solutions of Eq. (4.1) and put $Y_{t}=X_{t}-Z_{t}$. Then for $y \in \mathscr{D}\left(A^{*}\right)$

$$
\left(Y_{t} y\right)(\omega)=\int_{0}^{t}\left(Y_{s} A^{*} y\right)(\omega) d s, \quad \text { a.e. }(m)
$$

Let $t$ be (temporarily) fixed, $0<t \leq t_{1}$, and define a c.s.p. $\left\{Z_{s}\right\}_{s \in[0, t]}$ by $Z_{s} z=$ $Y_{s} T^{*}(t-s) z, z \in B_{1}^{*}$. Since $Y_{s}$ is uniformly square-integrable and $Y_{s} z$ is continuous in mean-square, it follows easily that $Z_{s}$ is uniformly square-integrable and $Z_{s} z$ is continuous in mean-square. For $y \in \mathscr{D}\left(A^{*}\right)$ it follows from Eq. (4.15) that $\left(Z_{0} y\right)(\omega)=0$, a.e. $(m)$.

Now require that $y \in \mathscr{D}\left(A^{* 2}\right)$ and $0<s<t$. Then for $\Delta$ sufficiently small,

$$
\begin{aligned}
\frac{1}{\Delta}\left(Z_{s} y-Z_{s-\Delta} y\right)= & \frac{1}{\Delta}\left(Y_{s}-Y_{s-\Delta}\right) T^{*}(t-s) y \\
& +\frac{1}{\Delta}\left[Y_{s-\Delta} T^{*}(t-s) y-Y_{s-\Delta} T^{*}(t-s+\Delta) y\right] .
\end{aligned}
$$

The first term on the right side above is equal to

$$
\frac{1}{\Delta} \int_{s}^{s+\Delta} Y_{u} A^{*} T^{*}(t-s) y d u
$$

by Eq. (4.15). It converges to $Y_{s} A^{*} T^{*}(t-s) y$ in mean-square (i.e., strongly in $\left.L_{2}(\Omega, Q, m)\right)$ as $\Delta \rightarrow 0$; in fact

$$
\begin{gathered}
\left\|\frac{1}{\Delta} \int_{s}^{s+\Delta} Y_{u} A^{*} T^{*}(t-s) y d u-Y_{s} A^{*} T^{*}(t-s) y\right\|_{L_{2}} \\
\quad \leq \frac{1}{\Delta} \int_{s}^{s+\Delta}\left\|\left(Y_{u}-Y_{s}\right) A^{*} T^{*}(t-s) y\right\|_{L_{2}} d u
\end{gathered}
$$

and $\left\|Y_{u} z\right\|_{L_{2}}$ is a continuous function.

The remaining term on the right side of Eq. (4.16) may be written

$$
Y_{s \sim \Delta}\left[\frac{1}{\Delta}\left(T^{*}(t-s) y-T^{*}(t-s+\Delta) y\right)\right] .
$$

For $\Delta \geq 0$, this equals

$$
Y_{s-\Delta}\left[-\frac{1}{\Delta} \int_{0}^{\Delta}(\Delta-\sigma) T^{*}(t-s+\sigma) A^{* 2} y d \sigma-T^{*}(t-s) A^{*} y\right] .
$$


Now $\left\|Y_{s-\Delta} z\right\|_{L_{2}}$ is bounded as $\Delta \rightarrow 0$, and

$$
\left\|\frac{1}{\Delta} \int_{0}^{\Delta}(\Delta-\sigma) T^{*}(t-s+\sigma) A^{* 2} y d \sigma\right\|_{L_{2}}=O(\Delta) ;
$$

so by uniform boundedness

$$
\left\|Y_{s-\Delta}\left[\frac{1}{\Delta} \int_{0}^{\Delta}(\Delta-\sigma) T^{*}(t-s+\sigma) A^{* 2} y d \sigma\right]\right\|_{L_{2}} \rightarrow 0
$$

as $\Delta \rightarrow 0$. Thus the second term on the right side of Eq. (4.6) converges in mean-square to $-Y_{s} T^{*}(t-s) A^{*} y$. For $\Delta<0$ a similar calculation yields the same result.

Thus, for all $y \in \mathscr{D}\left(A^{* 2}\right)$,

$$
\lim _{\Delta \rightarrow 0}\left\|\frac{1}{\Delta}\left(Z_{s} y-Z_{s-\Delta} y\right)\right\|_{L_{2}}=0 \quad 0<s<t .
$$

This implies that the real-valued function $\left\|Z_{s} y\right\|_{L_{2}}$ has derivative equal to zero for $0<s<t$. Since $\left\|Z_{s} y\right\|_{L_{2}}$ is a continuous function of $s$ for $0 \leq s \leq t$, it follows that it is constant on $[0, t]$ and hence equal to zero. In particular $\left\|Y_{t} y\right\|_{L_{2}}=\left\|Z_{t} y\right\|_{L_{2}}=0$. This has been shown for $0<t<t_{1}$, but by continuity it holds at $t=0$ and $t=t_{1}$ also. Since $\mathscr{Q}\left(A^{* 2}\right)$ is dense in $B_{1}^{*}$ and $Y_{t}$ is uniformly square-integrable, we have $\left\|Y_{t} y\right\|_{L_{2}}=0$ for all $y \in B_{1}^{*}$. Finally, the separability of the process $\left\{Y_{t} y\right\}_{t \in\left[0, t_{1}\right]}$ implies that

$$
m\left\{\sup _{t \in\left[0, t_{1}\right]}\left|Y_{t} y\right|<0\right\}=0 .
$$

We apply the theorem to an example of a stochastic differential equation in a Banach space $L_{p}, 1<p<2$. This choice of $B$ and $B_{1}$ makes the example easy because the injection $F$ is self-evident.

Example 4.1. Consider the weak stochastic differential equation in real $L_{p}$, $1<p<2$, for the classical problem of heat conduction in a thin ring with the addition of a "white-noise" forcing function. The equation is

$$
U_{t} y=U_{0} y+\int_{0}^{t} U_{s}\left(A^{*} y\right) d s+W_{t} y,
$$

$y \in \mathscr{D}\left(A^{*}\right)$, where $A$ is the infinitesimal generator of the semigroup involved. Thus the $\Phi(s, \omega)$ of Eq. (4.1) is taken to be identically one; however it would make little difference in what is to follow if it were chosen arbitrarily.

We suppose the ring has radius one, so that the function spaces involved are for functions on the unit circle. We write $L_{p}$ for real $L_{p}[0,2 \pi], L_{q}$ for real $L_{q}[0,2 \pi]$ where $q$ is fixed by $q^{-1}+p^{-1}=1$, and $A C_{2 \pi}$ for the space of real functions absolutely continuous on the circle. Since $p<2<q$ there is a natural 
injection $G: L_{q} \rightarrow L_{2}$ and a natural injection $F: L_{2} \rightarrow L_{p}$. The spaces $B_{1}$ and $B$ used in the specification of Eq. (4.1) are both taken to be $L_{p} ; B_{1}^{*}$ and $B^{*}$ are then identified with $L_{q}$.

The operator $A$ is the closed linear operator in $L_{p}$ defined by $A f=f^{\prime \prime}$ with domain $\mathscr{D}(A)=\left\{f \in L_{p}: f, f^{\prime} \in A C_{2 \pi}\right.$ and $\left.f^{\prime \prime} \in L_{p}\right\}$. It generates a semigroup $\{T(t) ; 0 \leq t<\infty\}$, and

$$
u\left(t ; f_{0}\right)=T(t) f_{0}
$$

is the unique solution to the homogeneous heat conduction equation in $L_{p}$. More precisely, it is the unique solution to the Cauchy problem: given $f_{0} \in L_{p}$, find a function $u(t)=u\left(t ; f_{0}\right)$ on $[0, \infty)$ to $L_{p}$ such that: (i) $u(t)$ is strongly continuously differentiable in $(0, \infty)$; (ii) for $t>0, u(t) \in \mathscr{D}(A)$ and $u^{\prime}(t)=A u(t)$; (iii) $s-\lim _{t \rightarrow 0^{+}} u\left(t, f_{0}\right)=f_{0}$ (see, for example, [5], Sect. 1.5.2). A formula for $(T(t) f)(\xi)$, $f \in L_{p}$, is

$$
(T(t) f)(\xi)=\sum_{k=-\infty}^{\infty} e^{-k^{2} t} \hat{f}(k) \phi_{k}(\xi), \quad t>0,
$$

where $\phi_{0}(\xi)=(2 \pi)^{-1 / 2} ; \phi_{k}(\xi)=\pi^{-1 / 2} \cos k \xi, k=1,2, \ldots ; \phi_{k}(\xi)=-\pi^{-1 / 2} \sin k \xi$, $k=-1,-2, \ldots$, and $\hat{f}(k)=\int_{0}^{2 \pi} f(\xi) \phi_{k}(\xi) d \xi$. It may readily be verified that $T(t)$ is symmetric and that $\left(T^{*}(t) g\right)(\xi), q \in L_{q}$, is given by a formula like that of Eq. (4.19), with $\hat{g}(k)$ replacing $\hat{f}(k)$. The generator of the $C_{0}$ semigroup $\left\{T^{*}(t)\right.$; $0 \leq t<\infty\}$ is $A^{*}$.

The c.W.p. $W_{t}$ is constructed as in Thm. 2.1. By identifying the Hilbert space $H=L_{2}$ with $H^{*}$, one has that the injection $G=F^{*}$. Define $C \in L\left(L_{q}, L_{p}\right)$ by $C=F F^{*} ; C$ is of course just the natural injection of $L_{q}$ into $L_{p}$. The o.n. set $\left\{\phi_{k}\right\}_{k=-\infty}^{\infty}$ introduced above lies in $\Re\left(F^{*}\right)$ and forms a basis for $\overline{\mathcal{R}\left(F^{*}\right)}$. Let $\left\{W_{t}^{i}\right.$, $t \geq 0\}_{i=-\infty}^{\infty}$ be a family of independent Wiener processes defined on a probability space $(\Omega, \mathbb{Q}, m)$. By Thm. 2.1 a c.W.p. is defined by

$$
\begin{aligned}
W_{t} y & =\sum_{i=-\infty}^{\infty} W_{t}^{i}\left(F \phi_{i}, y\right)_{L_{p}}=\sum_{i=-\infty}^{\infty} W_{t}^{i}\left(\phi_{i}, y\right)_{H} \\
& =\sum_{i=-\infty}^{\infty} W_{t}^{i} \hat{y}(i), \quad y \in L_{q},
\end{aligned}
$$

with covariance operators $t C$. By Lemma 2.2 there is a family $\left\{Q_{t}\right\}_{t \geq 0}$ of increasing sub- $\sigma$ algebras of $\mathscr{Q}$ such that $W_{t} y$ is nonanticipative with respect to $\mathbb{Q}_{t}$ for all $y \in L_{q}$.

If $U_{0}$ satisfies condition (iv) of Thm. 4.2, then by that theorem, the cylindrical process

$$
U_{t}=T(t) U_{0}+(w) \int_{0}^{t} T(t-s) d W_{s}
$$


gives an admissible solution to Eq. (4.17). Thus, for all $y \in \mathscr{D}\left(A^{*}\right)$,

$$
\begin{aligned}
U_{t} y & =U_{0}\left(T^{*}(t) y\right)+\sum_{k=-\infty}^{\infty} \int_{0}^{t}\left(T(t-s) F \phi_{k}, y\right) d W_{s}^{k} \\
& =U_{0}\left(\sum_{k=-\infty}^{\infty} e^{-k t^{2}} \hat{y}(k) \phi_{k}\right)+\sum_{k=-\infty}^{\infty} \int_{0}^{t}\left(T(t-s) \phi_{k}, y\right)_{L_{p}} d W_{s}^{k} \\
& =\sum_{k=-\infty}^{\infty} e^{-k t^{2}} \hat{y}(k) U_{0}\left(\phi_{k}\right)+\sum_{k=-\infty}^{\infty} \hat{y}(k) \int_{0}^{t} e^{-k^{2}(t-s)} d W^{k} s .
\end{aligned}
$$

\section{References}

1. Balakrishnan AV (1976) Applied functional analysis. Springer-Verlag, New York

2. Baxendale P (1974) Gaussian measures on function spaces. American J Math 98:891-952

3. Bensoussan A (1971) Filtrage optimale des systèmes linéaire. Dunod, Paris

4. Berman $N$ (1981) Stochastic integration in Banach spaces and applications. PhD thesis. The University of Michigan, Ann Arbor

5. Butzer PL, Berens H (1967) Semigroups of operators and approximation. Springer-Verlag, Berlin

6. Curtain RF, Falb PL (1971) Stochastic differential equations in Hilbert spaces. J Diff Eq 10:412-430

7. Curtain RF, Pritchard AJ (1978) Infinite dimensional linear system theory. Lecture notes in control and information systems 8 . Springer-Verlag, Berlin

8. Doob JL (1953) Stochastic processes. John Wiley and Sons, New York

9. Gross L (1965) Abstract Wiener spaces. In: Proc fifth Berkeley symp, Math Stat and Prob vol 2, part 2:31-42

10. Ito K, Nisio M (1968) On the convergence of sums of independent Banach space valued random variables. Osaka Math J 5:35-48

11. Kuo HH (1972) Stochastic integrals in abstract Wiener space. Pac J Math 41:469-483

12. Kuo HH (1973) Differential and stochastic equations in abstract Wiener space. J Func Anal $12: 246-256$

13. Lipster RS, Shiryayev AN (1977) Statistics of Random Processes I. Springer-Verlag, New York

14. Loève M (1977) Probability theory, 4th ed. Springer-Verlag, New York

15. Metivier M (1974) Stochastic integral with respect to processes with values in a reflexive Banach space. Th Prob Appl 19:758-787

16. Metivier M, Pellaumail J (1980) Stochastic integration. Academic Press, New York

17. Metivier M, Pistone G (1975) Une formule d'isometrie pour l'intégral stochastique Hilbertienne et équations d'evolution linéaire stochastique. $Z$ Wahr 33:1-18

18. Metivier M, Pistone G (1976) Sur une equation d'evolution stochastique. Bull Soc Math France 104:65-85 